\title{
Multiline Distance Minimization: A Visualized Many-Objective Test Problem Suite
}

\author{
Miqing Li, Crina Grosan, Shengxiang Yang, Senior Member, IEEE, Xiaohui Liu, and Xin Yao, Fellow, IEEE
}

\begin{abstract}
Studying the search behavior of evolutionary many-objective optimization is an important, but challenging issue. Existing studies rely mainly on the use of performance indicators which, however, not only encounter increasing difficulties with the number of objectives, but also fail to provide the visual information of the evolutionary search. In this paper, we propose a class of scalable test problems, called multiline distance minimization problem (ML-DMP), which are used to visually examine the behavior of many-objective search. Two key characteristics of the ML-DMP problem are: 1) its Pareto optimal solutions lie in a regular polygon in the 2-D decision space and 2) these solutions are similar (in the sense of Euclidean geometry) to their images in the high-dimensional objective space. This allows a straightforward understanding of the distribution of the objective vector set (e.g., its uniformity and coverage over the Pareto front) via observing the solution set in the 2-D decision space. Fifteen well-established algorithms have been investigated on three types of ten ML-DMP problem instances. Weakness has been revealed across classic multiobjective algorithms (such as Pareto-based, decomposition-based, and indicator-based algorithms) and even state-of-the-art algorithms designed especially for many-objective optimization. This, together with some interesting observations from the experimental studies, suggests that the proposed ML-DMP may also be used as a benchmark function to challenge the search ability of optimization algorithms.
\end{abstract}

Index Terms-Evolutionary algorithms, many-objective optimization, search behavior examination, test problems, visualization.

Manuscript received March 15, 2016; revised July 24, 2016 and November 7, 2016; accepted January 4, 2017. Date of publication January 18, 2017; date of current version January 26, 2018. This work was supported in part by the Engineering and Physical Sciences Research Council of U.K under Grant EP/K001523/1, Grant EP/K001310/1 and Grant EP/J017515/1, and in part by the National Natural Science Foundation of China under Grant 61403326, Grant 61329302, and Grant 61673331. The work of X. Yao was supported by the Royal Society Wolfson Research Merit Award.

$\mathrm{M}$. $\mathrm{Li}$ and $\mathrm{X}$. Yao are with the Centre of Excellence for Research in Computational Intelligence and Applications, School of Computer Science, University of Birmingham, Birmingham, B15 2TT, U.K. (e-mail: limitsing@gmail.com; x.yao@cs.bham.ac.uk).

C. Grosan is with the Department of Computer Science, Brunel University London, Uxbridge, UB8 3PH, U.K., and also with the Department of Computer Science, Babeş-Bolyai University, RO-400028 Cluj Napoca, Romania (e-mail: crina.grosan@brunel.ac.uk).

S. Yang is with the School of Computer Science and Informatics, De Montfort University, Leicester, LE1 9BH, U.K. (e-mail: syang@dmu.ac.uk).

$\mathrm{X}$. Liu is with the Department of Computer Science, Brunel University London, Uxbridge, UB8 3PH, U.K. (e-mail: xiaohui.liu@brunel.ac.uk).

This paper has supplementary downloadable multimedia material available at http://ieeexplore.ieee.org provided by the authors.

Digital Object Identifier 10.1109/TEVC.2017.2655451

\section{INTRODUCTION}

$\mathbf{E}$ XAMINATION of the search behavior of algorithms is an important issue in evolutionary optimization. It can help understand the characteristics of an evolutionary algorithm (e.g., knowing which kind of problems the algorithm may be appropriate for), facilitate its improvement, and also make a comparison between different algorithms.

However, search behavior examination can be challenging in the context of evolutionary multiobjective optimization (EMO). For a multiobjective optimization problem (MOP), there is often no single optimal solution (point) but rather a set of Pareto optimal solutions (Pareto optimal region). We may need to consider not only the convergence of the evolutionary population to these optimal solutions but also the representativeness of the population to the whole optimal region. This becomes even more difficult when an MOP has four or more objectives, usually called a many-objective optimization problem [1]-[3]. In many-objective optimization, the observation of the evolutionary population by the scatter plot, which is the predominating, most effective visualization method in bi- and tri-objective cases, becomes difficult to comprehend [4]-[8].

In the EMO community, there exist several test problem suites available for many-objective optimization. Among these, DTLZ [9] and WFG [10] are representations of continuous problem suites, and knapsack [11], traveling salesman problem [12] and multiobjective NK-landscapes [13] are representations of discrete ones. Recently, researchers have also presented several new MOPs for many-objective optimization [14]-[16]. The above test problems have their own characteristics, and some of them have been widely used to examine the performance of many-objective evolutionary algorithms [17]-[26]. In the performance examination, an algorithm is called on a test problem and then returns a set of solutions with high dimensions.

How to assess such a solution set is not a trivial task. The basic way is to resort to performance indicators. Unfortunately, there is no performance indicator that is able to fully reflect the search behavior of evolutionary algorithms. On the one hand, it is challenging to find (or design) a performance indicator suited well to many-objective optimization, as a result of growing difficulties with the number of objectives, such as the requirement of time and space complexity, ineffectiveness of the Pareto dominance criterion, sensitivity of the parameter settings, and inaccuracy of the Pareto front's substitution. Many performance indicators that are designed in principle for any number of objectives 
may be invalid or infeasible in practice in many-objective optimization [27].

On the other hand, one performance indicator only examines one specific aspect of algorithms' behavior. Even those performance indicators that aim to examine the same aspect of the population performance also have their own preference. For example, two commonly used indicators, inverted generational distance (IGD) [28] and hypervolume (HV) [11], both of which provide a combined information of convergence and diversity of the population, can bring inconsistent assessment results [29]-[31]. For two populations being of same convergence, IGD, which is calculated on the basis of uniformly distributed points along the Pareto front, prefers the one having uniformly distributed individuals, while $\mathrm{HV}$, which is typically influenced more by the boundary individuals, has a bias toward the one having good extensity.

More importantly, performance indicators cannot provide the visual information of the evolutionary search. This matters, especially for researchers and practitioners with the real-world application background who typically have no expertise in the EMO performance assessment-it could be hard for them to understand the behavior of EMO algorithms only on the basis of the returned indicator values.

Recently, EMO researchers introduced a class of test problems (called the multipoint distance minimization problem (MP-DMP) ${ }^{1}$ for visual examination of the search behavior of multiobjective optimizers. As its name suggests, the MPDMP problem is to simultaneously minimize the distance of a point to a prespecified set (or several prespecified sets) of target points. One key characteristic of MP-DMP is its Pareto optimal region in the decision space is typically a 2-D manifold (regardless of the dimensionality of its objective vectors and decision variables). This naturally allows a direct observation of the search behavior of EMO algorithms, e.g., the convergence of their population to the Pareto optimal solutions and the coverage of the population over the optimal region.

Over the last decade, the MP-DMP problem and its variants have gained increasing attention in the evolutionary multiobjective (esp. many-objective) optimization area. Köppen and Yoshida [33] constructed a simple MP-DMP instance which minimizes the Euclidean distance of a point to a set of target points in a 2-D space. This leads to the Pareto optimal solutions residing in the convex polygon formed by the target points. Rudolph et al. [38] introduced a variant of MP-DMP whose Pareto optimal solutions are distributed in multiple symmetrical regions in order to investigate if EMO algorithms are capable of detecting and preserving equivalent Pareto subsets. Schütze et al. [39] and Singh et al. [40] used the MP-DMP problem to help understand the characteristics of many-objective optimization, analytically and empirically, respectively. Ishibuchi et al. [41] generalized the MP-DMP problem and introduced multiple Pareto optimal polygons with same [41] or different shapes [42]. Later on, they examined the behavior of EMO algorithms on the MP-DMP

\footnotetext{
${ }^{1}$ The MP-DMP has different names or abbreviations in the literature (such as the Pareto box problem [32], $P^{*}$ problem [33], distance minimization problem [34], DMP [35], and M-DMP [36]). For the contrast of the work presented in this paper, we abbreviate it as MP-DMP here [37].
}

problem with an arbitrary number of decision variables [34], and also further generalized this problem by specifying reference points on a plane in the high-dimensional decision space [43]. Very recently, Zille and Mostaghim [35] used the Manhattan distance measure in MP-DMPs and found that this can drastically change the problem's property and difficulty. Xu et al. [36] proposed a systematic procedure to identify Pareto optimal solutions of the MP-DMP under the Manhattan distance measure and also gave a theoretical proof of their Pareto optimality. Fieldsend [44] embedded dominance resistance regions into the MP-DMP problem and demonstrated that Pareto-based approach can be fragile to dominance resistance points. Overall, the MP-DMP problems present a good alternative for researchers to understand the behavior of multiobjective search. Consequently, they have been frequently used to visually compare many-objective optimizers in recent studies [45]-[47].

However, one weakness of the MP-DMP problem is its inability to facilitate examination of the search behavior in the objective space. There is no explicit (geometric) similarity relationship between decision variables' distribution and that of objective vectors. Even when a set of objective vectors are distributed perfectly over the Pareto front, we cannot know this fact via observing the corresponding solution variables in the decision space.

As the first attempt to solve the above issue, we recently presented a four-objective test problem whose Pareto optimal solutions in the decision space are similar (in the sense of Euclidean geometry) to their images in the objective space [48]. This therefore allows a straightforward understanding of the behavior of objective vectors, e.g., their uniformity and coverage over the Pareto front. However, to comply to the geometric similarity between the Pareto optimal solutions and their objective images, the presented problem fixes its objective dimensionality to four. This makes it impossible to examine the search behavior of EMO algorithms in a higher-dimensional objective space.

In this paper, we significantly extend our previous work in [48] and propose a class of test problems [called the multiline distance minimization problem (ML-DMP)] whose objective dimensionality is changeable. In contrast to the MPDMP which minimizes the distance of a point to a set of target points, the proposed ML-DMP minimizes the distance of a point to a set of target lines. Two key characteristics of the ML-DMP are that its Pareto optimal solutions: 1) lie in a regular polygon in the 2-D decision space and 2) are similar (in the sense of Euclidean geometry) to their images in the high-dimensional objective space. In addition to these, the ML-DMP has the following properties.

1) It is scalable with respect to the number of objectivesits objective dimensionality can be set by the user freely.

2) Its difficulty level is adjustable, which allows a viable examination of diverse search abilities of EMO algorithms.

3) It provides an interesting dominance structure which varies with the number of objectives, e.g., for the fourobjective instance there exist some areas dominated only by one line segment and for the five-objective one there exist some areas dominated only by one particular point. 


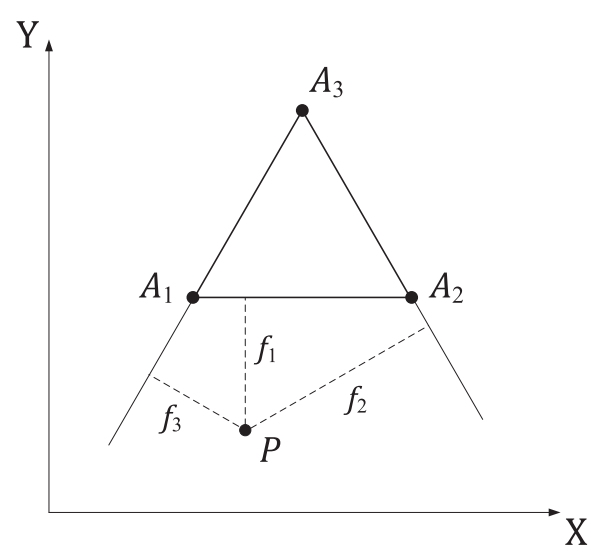

Fig. 1. Tri-objective ML-DMP instance where $A_{1}-A_{3}$ are the three vertexes of the regular triangle.

This paper conducts a theoretical analysis of the geometric similarity of the Pareto optimal solutions and also of their optimality in the polygon as to the given search space. For experimental examination, this paper considers ten instances of the ML-DMP problem with three, four, five, and ten objectives and investigates the search behavior of 15 well-established algorithms on these instances. This investigation provides a visual understanding of the search behavior of EMO algorithms on different objective dimensionality and varying search space.

The rest of this paper is structured as follows. Section II describes the proposed ML-DMP and this includes an analysis of the problem's geometric similarity and Pareto optimality. Section III introduces experimental design. Section IV is devoted to experimental results. Finally, Section V draws the conclusions and gives possible lines of future work.

\section{Multiline Distance Minimization Problem}

The ML-DMP considers a 2-D decision space. For any point $P=(x, y)$ in this space, the ML-DMP calculates the Euclidean distance from $P$ to a set of $m$ target straight lines, each of which passes through an edge of the given regular polygon with $m$ vertexes $\left(A_{1}, A_{2}, \ldots, A_{m}\right)$, where $m \geq 3$. The goal in the ML-DMP is to optimize these $m$ distance values simultaneously. Fig. 1 gives a tri-objective ML-DMP instance. $A_{1}-A_{3}$ are the three vertexes of a regular triangle, and $\overleftrightarrow{A_{1} A_{2}}$, $\overleftrightarrow{A_{2} A_{3}}$, and $\overleftrightarrow{A_{3} A_{1}}$ are the three target lines passing through the three edges of the triangle. Thus, the objective vector of a point $P$ is $\left(f_{1}, f_{2}, f_{3}\right)=\left(d\left(P, \overleftrightarrow{A_{1} A_{2}}\right), d\left(P, \overleftrightarrow{A_{2} A_{3}}\right), d\left(P, \overleftrightarrow{A_{3} A_{1}}\right)\right)$ where $d\left(P, \overleftrightarrow{A_{i} A_{j}}\right)$ denotes the Euclidean distance from point $P$ to straight line $\overleftrightarrow{A_{i} A_{j}}$.

It is clear that there does not exist a single point $P$ on the decision space that can reach minimal value for all the objectives. For the tri- or four-objective ML-DMP, the Pareto optimal region is their corresponding regular polygon. But this may not be the case for the ML-DMP with five or more objectives. The identification of the Pareto

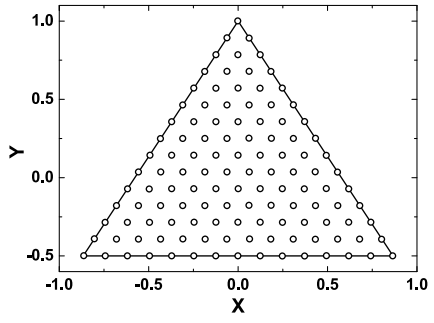

(a)

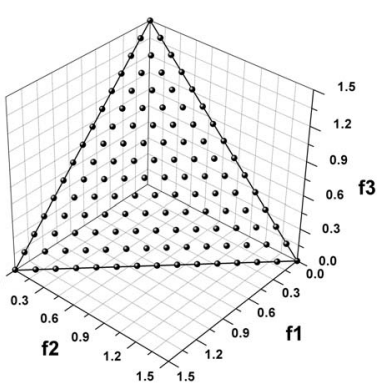

(b)
Fig. 2. Illustration of geometric similarity of the ML-DMP on a tri-objective instance, where a set of uniformly-distributed points over the regular triangle in the decision space lead to a set of uniformly-distributed objective vectors. (a) Decision space. (b) Objective space.

optimal solutions of the ML-DMP will be presented in Section II-B.

\section{A. Geometric Similarity of the ML-DMP}

An important characteristic of the ML-DMP is that the points in the regular polygon (including the boundaries) and their objective images are similar in the sense of Euclidean geometry. In other words, the ratio of the distance between any two points in the polygon to the distance between their corresponding objective vectors is a constant. Fig. 2 illustrates the geometric similarity between the polygon points and their images on a tri-objective ML-DMP. Next, we give the definition of the geometric similarity for an ML-DMP with any number of objectives.

Theorem 1: For an ML-DMP problem, the Euclidean distance between any two solutions that lie inside the regular polygon (including the boundaries) is equal to the Euclidean distance between their objective images multiplied by a constant. Formally, for any two interior solutions $P_{1}\left(x_{1}, y_{1}\right)$ and $P_{2}\left(x_{2}, y_{2}\right)$ of the polygon of an ML-DMP problem $F=$ $\left(f_{1}, f_{2}, \ldots, f_{m}\right)$, we have

$$
\left\|P_{1}-P_{2}\right\|=k\left\|F\left(P_{1}\right)-F\left(P_{2}\right)\right\|
$$

which can be rewritten as

$$
\sqrt{\left(x_{1}-x_{2}\right)^{2}-\left(y_{1}-y 2\right)^{2}}=k \sqrt{\sum_{i=1}^{m}\left(f_{i}\left(P_{1}\right)-f_{i}\left(P_{2}\right)\right)^{2}} .
$$

Proof: Let us consider the notations as in Fig. 3. Without loss of generality, it can be supposed that one edge of the polygon (for instance $\overline{A_{1} A_{2}}$ ) is parallel with $x$-axis and $P_{1}$ and $P_{2}$ are two points inside the polygon. Let us denote by $\theta$ the angle between the line $\overleftrightarrow{P_{1} P_{2}}$ and $x$-axis. Then

$$
\begin{aligned}
f_{1}\left(P_{1}\right)-f_{1}\left(P_{2}\right) & =d\left(P_{1}, \overleftrightarrow{A_{1} A_{2}}\right)-d\left(P_{2}, \overleftrightarrow{A_{1} A_{2}}\right) \\
& =l \sin \theta
\end{aligned}
$$

where $l$ is the Euclidean distance between $P_{1}$ and $P_{2}$ (i.e., $\left.\left\|P_{1}-P_{2}\right\|=l\right)$. 


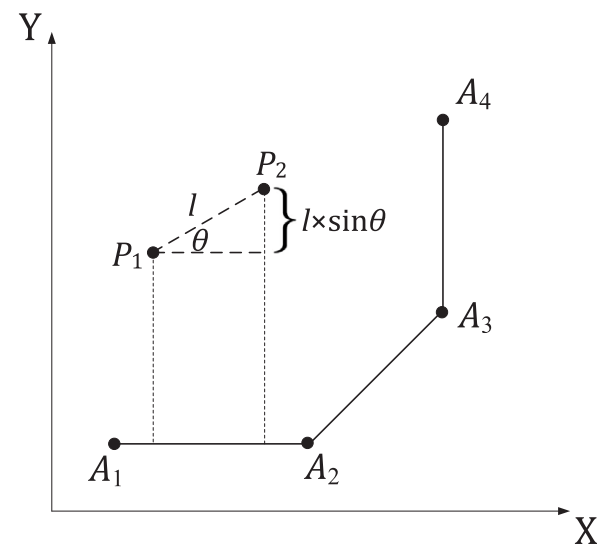

Fig. 3. Illustration for the proof of Theorem 1.

Since the polygon is regular, the same property holds for the edge $\overleftrightarrow{A_{2} A_{3}}$, with the difference that the angle between $\overleftrightarrow{A_{2} A_{3}}$ and $x$-axis is $2 \pi / m$. Thus, we have

$$
\begin{aligned}
f_{2}\left(P_{1}\right)-f_{2}\left(P_{2}\right) & =d\left(P_{1}, \overleftrightarrow{A_{2} A_{3}}\right)-d\left(P_{2}, \overleftrightarrow{A_{2} A_{3}}\right) \\
& =l \sin (\theta-2 \pi / m) .
\end{aligned}
$$

This can be visualized as rotating the coordinate system with $2 \pi / m$ degrees, when the angle between the line $\overleftrightarrow{P_{1} P_{2}}$ and $x$-axis in the new coordinate system is $\theta-2 \pi / m$. Now we further have

$$
\left\|F\left(P_{1}\right)-F\left(P_{2}\right)\right\|=\sqrt{\sum_{i=0}^{m-1}\left(l \sin \left(\theta-\frac{2 \pi i}{m}\right)\right)^{2}} .
$$

Using the relation $\sin ^{2} \theta=(1-\cos 2 \theta) / 2$, the above equation can be written as

$$
\begin{aligned}
\left\|F\left(P_{1}\right)-F\left(P_{2}\right)\right\| & =l \sqrt{\sum_{i=0}^{m-1} \frac{1-\cos \left(2 \theta-\frac{4 \pi i}{m}\right)}{2}} \\
& =\frac{l}{\sqrt{2}} \sqrt{m-\sum_{i=0}^{m-1} \cos \left(2 \theta-\frac{4 \pi i}{m}\right)} .
\end{aligned}
$$

If we change the index of the sum from $i=0,1, \ldots, m-$ 1 to $i=0,1, \ldots, m-1, m$ and use the relation $\cos (2 \theta-$ $4 \pi m / m)=\cos 2 \theta$, the equation can be expressed as

$$
\begin{aligned}
& \left\|F\left(P_{1}\right)-F\left(P_{2}\right)\right\| \\
= & \frac{l}{\sqrt{2}}\left(m+\cos 2 \theta-\sum_{i=0}^{m} \cos \left(2 \theta-\frac{4 \pi i}{m}\right)\right)^{\frac{1}{2}} \\
= & \frac{l}{2}\left(2 m+2 \cos 2 \theta-2 \sum_{i=0}^{m} \cos \left(2 \theta-\frac{4 \pi i}{m}\right)\right)^{\frac{1}{2}} \\
= & \frac{l}{2}\left(2 m+2 \cos 2 \theta-\sum_{i=0}^{m}\left(\cos \left(2 \theta-\frac{4 \pi i}{m}\right)\right.\right. \\
& \left.\left.\quad+\cos \left(2 \theta-\frac{4 \pi(m-i)}{m}\right)\right)\right)^{\frac{1}{2}} .
\end{aligned}
$$

According to the relation $\cos \alpha+\cos \beta=2 \cos [(\alpha+\beta) / 2]$. $\cos [(\alpha-\beta) / 2]$, the equation can be further expressed as

$$
\begin{aligned}
& \left\|F\left(P_{1}\right)-F\left(P_{2}\right)\right\| \\
& =\frac{l}{2}(2 m+2 \cos 2 \theta \\
& -\sum_{i=0}^{m} 2 \cos \frac{\left(2 \theta-\frac{4 \pi i}{m}\right)+\left(2 \theta-\frac{4 \pi(m-i)}{m}\right)}{2} \\
& \left.\times \cos \frac{\left(2 \theta-\frac{4 \pi i}{m}\right)-\left(2 \theta-\frac{4 \pi(m-i)}{m}\right)}{2}\right)^{\frac{1}{2}} \\
& =\frac{l}{2}\left(2 m+2 \cos 2 \theta-2 \sum_{i=0}^{m} \cos \left(2 \theta-\frac{2 \pi m}{m}\right)\right. \\
& \left.\times \cos \frac{2 \pi(m-2 i)}{m}\right)^{\frac{1}{2}} \\
& =\frac{l}{2}\left(2 m+2 \cos 2 \theta-2 \cos 2 \theta \sum_{i=0}^{m} \cos \left(2 \pi-\frac{4 \pi i}{m}\right)\right)^{\frac{1}{2}} \\
& =\frac{l}{2}\left(2 m+2 \cos 2 \theta-2 \cos 2 \theta \sum_{i=0}^{m} \cos \frac{4 \pi i}{m}\right)^{\frac{1}{2}} \\
& =\frac{l}{2}(2 m+2 \cos 2 \theta \\
& \left.-2 \cos 2 \theta\left(\sum_{i=0}^{m-1} \cos \frac{4 \pi i}{m}+\cos \frac{4 \pi m}{m}\right)\right)^{\frac{1}{2}} \\
& =\frac{l}{2}\left(2 m+2 \cos 2 \theta-2 \cos 2 \theta\left(\sum_{i=0}^{m-1} \cos \frac{4 \pi i}{m}+1\right)\right)^{\frac{1}{2}} \\
& =\frac{l}{2}\left(2 m-2 \cos 2 \theta \sum_{i=0}^{m-1} \cos \frac{4 \pi i}{m}\right)^{\frac{1}{2}} \text {. }
\end{aligned}
$$

Now we simplify the above equation. Let us consider the complex number $\omega=\cos (4 \pi / m)+i \sin (4 \pi / m)$. Then $\omega^{x}=\cos (4 \pi x / m)+i \sin (4 \pi x / m)$ and $\omega^{m}=\cos (4 \pi m / m)+$ $i \sin (4 \pi m / m)=\cos 4 \pi+i \sin 4 \pi=1$. We know that $\omega^{m}-1=0$. Thus $(\omega-1)\left(\omega^{m-1}+\omega^{m-2}+\cdots+\omega+1\right)=0$. Since $\omega \neq 1$, it holds $\omega^{m-1}+\omega^{m-2}+\cdots+\omega+1=0$, which means that $\sum_{i=0}^{m-1}(\cos (4 \pi i / m)+i \sin (4 \pi i / m))=0$. This indicates that both real and imaginary parts equal 0 . Thus

$$
\sum_{i=0}^{m-1} \cos \frac{4 \pi i}{m}=0 .
$$

If we now go back to (5), we have

$$
\begin{aligned}
& \left\|F\left(P_{1}\right)-F\left(P_{2}\right)\right\| \\
& =\frac{l}{2}\left(2 m-2 \cos 2 \theta \sum_{i=0}^{m-1} \cos \frac{4 \pi i}{m}\right)^{\frac{1}{2}}=\frac{l}{2} \sqrt{2 m}=l \sqrt{\frac{m}{2}} .
\end{aligned}
$$


Since $\left\|P_{1}-P_{2}\right\|=l$, we finally have

$$
\left\|P_{1}-P_{2}\right\|=\sqrt{\frac{2}{m}}\left\|F\left(P_{1}\right)-F\left(P_{2}\right)\right\|
$$

where $m$ is the problem's objective dimensionality. This completes the proof of Theorem 1 .

Note that the polygon of the ML-DMP should be regular; otherwise, this theorem does not hold.

\section{B. Pareto Optimality of the $M L-D M P$}

To consider the Pareto optimality of the ML-DMP, let us first recall several well-known concepts in multiobjective optimization: Pareto dominance, Pareto optimality, Pareto optimal set and Pareto front. Without loss of generality, we consider the minimization MOP here.

Definition 1 (Pareto Dominance): For an MOP $F(P)=$ $\left(f_{1}(P), f_{2}(P), \ldots, f_{m}(P)\right)$, let $P_{1}$ and $P_{2}$ be two feasible solutions (denoted as $P_{1}, P_{2} \in \Omega$ ). $P_{1}$ is said to Pareto dominate $P_{2}$ (denoted as $P_{1} \prec P_{2}$ ), if and only if

$$
\begin{aligned}
& \forall i \in(1,2, \ldots, m): f_{i}\left(P_{1}\right) \leq f_{i}\left(P_{2}\right) \wedge \\
& \exists j \in(1,2, \ldots, m): f_{j}\left(P_{1}\right)<f_{j}\left(P_{2}\right) .
\end{aligned}
$$

On the basis of the concept of Pareto dominance, the Pareto optimality and Pareto optimal set (Pareto front) can be defined as follows.

Definition 2 (Pareto Optimality): A solution $P^{*} \in \Omega$ is said to be Pareto optimal if there is no $P \in \Omega, P \prec P^{*}$.

Definition 3 (Pareto Optimal Set and Pareto Front): The Pareto optimal set is defined as the set of all Pareto optimal solutions, and the Pareto front is the set of their corresponding images in the objective space.

Next, we discuss the Pareto optimal solutions of the ML-DMP problem.

Theorem 2: For an ML-DMP $\left(\Omega=\mathbb{R}^{2}\right)$ with a regular polygon of $m$ vertexes $\left(A_{1}, A_{2}, \ldots, A_{m}\right)$, points inside the polygon (including the boundary points) are the Pareto optimal solutions. In other words, for any point in the polygon, there is no point $\in \mathbb{R}^{2}$ that dominates it.

Proof: See Section I in the supplementary material.

This theorem indicates that all points inside the polygon are the Pareto optimal solutions. However, these points may not be the sole Pareto optimal solutions of the problem. That is, there may exist some points outside the polygon that are not dominated by these points.

Consider a five-objective ML-DMP in Fig. 4, where $A_{1}-A_{5}$ are the five vertexes of the regular pentagon. Point $O$ is the intersection point of the two target lines $\overleftrightarrow{A_{1} A_{2}}$ and $\overleftrightarrow{A_{4} A_{3}}$, and $A_{2}^{\prime}$ and $A_{3}^{\prime}$ are the symmetric points of $A_{2}$ and $A_{3}$ with respect to point $O$, respectively. As to the two objectives of target lines $\overleftrightarrow{A_{1} A_{2}}$ and $\overleftrightarrow{A_{4} A_{3}}$, we have that there is no point inside the pentagon that is better than any point in the region bounded by points $A_{2}, A_{3}^{\prime}, A_{2}^{\prime}$, and $A_{3}$ (denoted as polygon $\overline{A_{2} A_{3}^{\prime} A_{2}^{\prime} A_{3} A_{2}}$ ). To see this, let us divide polygon $\overline{A_{2} A_{3}^{\prime} A_{2}^{\prime} A_{3} A_{2}}$ into four triangles: 1) $\overline{A_{2} O A_{3} A_{2}}$; 2) $\overline{A_{2}^{\prime} O A_{3}^{\prime} A_{2}^{\prime}}$; 3) $\overline{A_{3} O A_{2}^{\prime} A_{3}}$; and 4) $\overline{A_{2} O A_{3}^{\prime} A_{2}}$. For triangle $\overline{\mathrm{A}_{2} \mathrm{OA}_{3} A_{2}}$, it is clear that its points are not dominated by the pentagon point with respect to the two objectives of target lines $\overleftrightarrow{A_{1} A_{2}}$ and $\overleftrightarrow{A_{4} A_{3}}$. This can be explained by the

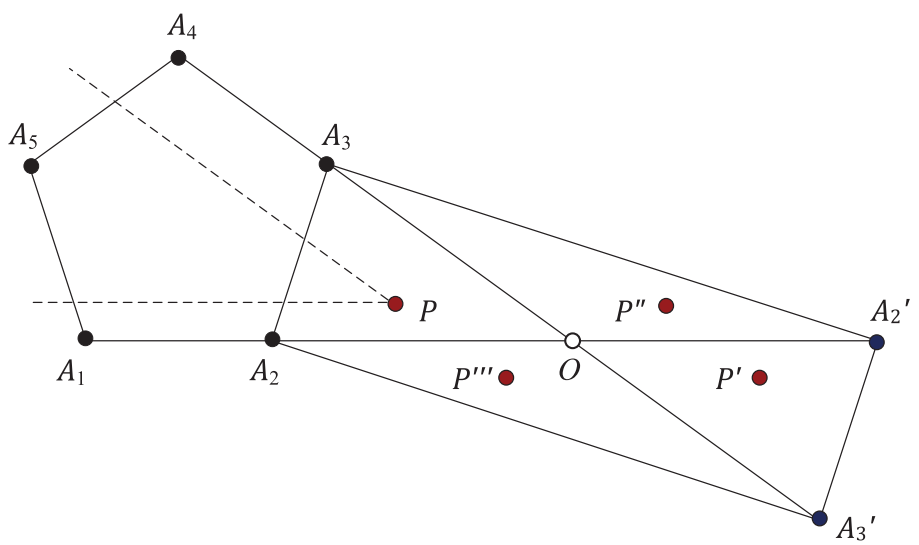

Fig. 4. Illustration of some areas outside the polygon being nondominated areas on a five-objective ML-DMP.

fact that for any point in triangle $\overline{A_{2} \mathrm{OA}_{3} A_{2}}$ (e.g., $P$ in Fig. 4), there is no intersection of the two areas of the regular pentagon: one is the area that is better than $P$ for target line $\overleftrightarrow{A_{1} A_{2}}$ and the other is the area that is better than $P$ for target line $\overleftrightarrow{A_{4} A_{3}}$. On the other hand, according to the structure properties of the polygon $\overline{A_{2} A_{3}^{\prime} A_{2}^{\prime} A_{3} A_{2}}$, it is not difficult to obtain that for any point in the other three triangles: 1) $\overline{A_{2}^{\prime} O A_{3}^{\prime} A_{2}^{\prime}}$; 2) $\overline{A_{3} O A_{2}^{\prime} A_{3}}$; and 3) $\overline{A_{2} O A_{3}^{\prime} A_{2}}$, there exists a corresponding point in triangle $\overline{\mathrm{A}_{2} \mathrm{OA}_{3} \mathrm{~A}_{2}}$ that has same distance to target lines $\overleftrightarrow{A_{1} A_{2}}$ and $\overleftrightarrow{A_{4} A_{3}}$ (i.e., same value on these two objectives). This includes that when a point is located on boundary lines $\overline{A_{3} A_{2}^{\prime}}, \overline{A_{2}^{\prime} A_{3}^{\prime}}$ or $\overline{A_{3}^{\prime} A_{2}}$, there exists a corresponding point on line $\overline{A_{2} A_{3}}$. So, for any point inside polygon $\overline{A_{2} A_{3}^{\prime} A_{2}^{\prime} A_{3} A_{2}}$ (excluding the boundary), there is no point in the regular pentagon (including the boundary) that is better than (or equal to) it with respect to both target lines $\overleftrightarrow{A_{1} A_{2}}$ and $\overleftrightarrow{A_{4} A_{3}}$.

The above discussions indicate that in an ML-DMP if two target lines intersect outside the regular polygon, there exist some areas whose points are nondominated with the interior points of the polygon. Apparently, such areas exist in an MLDMP with five or more objectives in view of the convexity of the considered polygon. However, according to Theorem 1, the geometric similarity holds only for the points inside the regular polygon. The Pareto optimal solutions that are located outside the polygon will affect this similarity property. To address this issue, we constrain some regions in the search space of the ML-DMP so that the points inside the regular polygon are the sole Pareto optimal solutions of the problem.

Formally, consider an $m$-objective ML-DMP with a regular polygon of vertexes $\left(A_{1}, A_{2}, \ldots, A_{m}\right)$. For any two target lines $\overleftrightarrow{A_{i-1} A_{i}}$ and $\overleftrightarrow{A_{n} A_{n+1}}$ (without loss of generality, assuming $i<n)$ that intersect one point $(O)$ outside the considered regular polygon, we can construct a polygon (denoted as $\left.\Phi_{A_{i-1} A_{i} A_{n} A_{n+1}}\right)$ bounded by a set of $2(n-i)+2$ line segments: $\overline{A_{i} A_{n}^{\prime}}, \overline{A_{n}^{\prime} A_{n-1}^{\prime}}, \ldots, \overline{A_{i+1}^{\prime} A_{i}^{\prime}}, \overline{A_{i}^{\prime} A_{n}}, \overline{A_{n} A_{n-1}}, \ldots, \overline{A_{i+1} A_{i}}$, where points $A_{i}^{\prime}, A_{i+1}^{\prime}, \ldots, A_{n-1}^{\prime}, A_{n}^{\prime}$ are symmetric points of $A_{i}, A_{i+1}, \ldots A_{n-1}, A_{n}$ with respect to central point $O$. We constrain the search space of the ML-DMP outside such polygons (but not including the boundary). Now we have the following theorem. 
Theorem 3: Considering an ML-DMP with a regular polygon of $m$ vertexes $\left(A_{1}, A_{2}, \ldots, A_{m}\right)$, the feasible region $\Omega=$ $\bar{\Phi} \wedge S$, where $\Phi$ is the union set of all the constrained polygons and $S$ is a 2-D rectangle space in $\mathbb{R}^{2}$ (i.e., the rectangle constraint defined by the marginal values of decision variables). Then, the points inside the regular polygon (including the boundary) are the sole Pareto optimal solutions of the ML-DMP.

Proof: See Section II in the supplementary material.

Note that the feasible region of the problem includes the boundary points of the constrained polygons, which are typically dominated by only one Pareto optimal point. This property can cause difficulty for EMO algorithms to converge. In addition, unlike MP-DMP where solutions far from the optimal polygon have poor values on all the objectives (as they are away from all the target vertexes of the polygon), in MLDMP solutions far from the optimal polygon will have the best (or near best) value on one of the objectives when they are located on (or around) one target line. Such solutions belong to so-called dominance resistant solutions [49] [i.e., the solutions with an (near) optimal value in at least one of the objectives but with quite poor values in the others], which many EMO algorithms have difficulty in getting rid of [49], [50]. Moreover, for an ML-DMP with an even number of objectives $(m=2 k$ where $k \geq 2$ ), there exist $k$ pairs of parallel target lines. Any point (outside the regular polygon) residing between a pair of parallel target lines is dominated by only a line segment parallel to these two lines. This property of the ML-DMP problem poses a great challenge for EMO algorithms which use Pareto dominance as the sole selection criterion in terms of convergence, typically leading to their populations trapped between these parallel lines.

\section{EXPERIMENTAL DESIGN}

\section{A. Three Types of ML-DMP Instances}

To systematically examine the search behavior of EMO algorithms in terms of convergence and diversity, three types of ML-DMP instances are considered. For all the instances, the center coordinates of the regular polygon (i.e., Pareto optimal region) are $(0,0)$ and the radius of the polygon (i.e., the distance of the vertexes to the center) is 1.0.

In type I, the search space of the ML-DMP is precisely the Pareto optimal region (i.e., the regular polygon). This allows us to solely understand the ability of EMO algorithms in maintaining diversity. The search space of type II is $[-100,100]^{2}$, which is used to examine the ability of algorithms in balancing convergence and diversity. In type III, the search space of the problem is extended hugely to $\left[-10^{10}, 10^{10}\right]^{2}$. This focuses on the examination of algorithms' ability in driving the population toward the optimal region.

Three-, four-, five-, and ten-objective ML-DMP problems are considered in the experimental studies. In the threeobjective ML-DMP, there are no parallel target lines and constrained areas. It is expected that EMO algorithms can relatively easily find the optimal polygon. The four- and fiveobjective ML-DMPs have parallel target lines and constrained areas, respectively, which present difficulties for Pareto-based algorithms to converge. The ten-objective problem has a lot of parallel target lines and constrained areas. This should provide a big challenge for EMO algorithms in guiding the population into the optimal region.

\section{B. Examined Algorithms}

Fifteen EMO algorithms are examined, including classic EMO algorithms (such as Pareto-based, decompositionbased, and indicator-based algorithms) and also those designed specially for many-objective optimization. Next, we briefly describe these algorithms.

1) Nondominated Sorting Genetic Algorithm II (NSGA-II) [51]: As one of the most popular EMO algorithms, NSGA-II is characterized as the Pareto nondominated sorting and crowding distance in its fitness assignment.

2) Strength Pareto Evolutionary Algorithm 2 (SPEA2) [52]: SPEA2 is also a prevalent Pareto-based algorithm, which uses a so-called fitness strength and the nearest neighbor technique to compare individuals during the evolutionary process.

3) Average Ranking (AR) [53]: AR is regarded as a good alternative in solving many-objective optimization problems [12]. It first ranks solutions in each objective and then sums up all the rank values to evaluate the solutions. However, due to a lack of diversity maintenance mechanism, AR often leads the population to converge into a subarea of the Pareto front [18], [64].

4) Indicator-Based Evolutionary Algorithm (IBEA) [54]: As the pioneer of indicator-based EMO algorithms, IBEA defines the optimization goal in terms of a binary performance measure and then utilizes this measure to guide the search. Two indicators, $I_{\epsilon+}$ and $I_{H V}$, were considered in IBEA. Here, $I_{\epsilon+}$ is used in our experimental studies.

5) $\epsilon$-Dominance Multiobjective Evolutionary Algorithm ( $\epsilon-M O E A)$ [55]: Using the $\epsilon$ dominance [65] to strengthen the selection pressure, $\epsilon$-MOEA has been found to be promising in many-objective optimization [19], [45]. The algorithm divides the objective space into many hyperboxes and allows each hyperbox at most one solution according to the $\epsilon$ dominance and the distance from solutions to the utopia point in the hyperbox.

6) S Metric Selection EMO Algorithm (SMS-EMOA) [56]: SMS-EMOA, like IBEA, is also an indicator-based algorithm. It combines the maximization of the HV contribution with the nondominated sorting. Despite having good performance in terms of both convergence and diversity, SMS-EMOA suffers from an exponentially increasing computational cost. In this paper, when the number of the problem's objectives reaches five, we approximately estimate the HV contribution of SMS-EMOA by the Monte Carlo sampling method used in [59].

7) Multiobjective Evolutionary Algorithm Based on Decomposition (MOEA/D) [57]: As one of the most well-known algorithms developed recently, MOEA/D converts a multiobjective problem into a set of scalar 
TABLE I

Parameter Setting And the Source of the Tested Algorithms

\begin{tabular}{l|l|l}
\hline Algorithm & Parameter(s) & Source \\
\hline NSGA-II [51] & & http://www.iitk.ac.in//kangal \\
\hline SPEA2 [52] & & http://www.tik.ee.ethz.ch/pisa \\
\hline AR [53] & & written by ourselves \\
\hline IBEA [54] & $\kappa=0.05$ & http://www.tik.ee.ethz.ch/pisa \\
\hline$\epsilon$-MOEA [55] & see Table II for the $\epsilon$ setting & http://www.iitk.ac.in/kangal \\
\hline SMS-EMOA [56] & & http://jmetal.sourceforge.net/index.html \\
\hline MOEA/D-TCH [57] & neighborhood size 10\% & http://dces.essex.ac.uk/staff/qzhang/ \\
\hline MOEA/D-PBI [57] & neighborhood size 10\%, penalty parameter 5.0 & http://dces.essex.ac.uk/staff/qzhang/ \\
\hline DMO [58] & & written by ourselves \\
\hline HypE [59] & sampling point 10,000 & http://www.tik.ee.ethz.ch/pisa \\
\hline GrEA [60] & grid division 20 & http://www.cs.bham.ac.uk/\%7Elimx \\
\hline Two_Arch2 [61] & $\kappa=0.05$ & http://www.cs.bham.ac.uk/\%7Exin \\
\hline AGE-II [62] & $\epsilon_{\text {grid }}=0.1$ & http://cs.adelaide.edu.au/\%7Emarkus \\
\hline NSGA-III [63] & & http://web.ntnu.edu.tw/\%7Etcchiang/publications/nsga3cpp/nsga3cpp \\
\hline SPEA2+SDE [46] & & http://www.cs.bham.ac.uk/\%7Elimx \\
\hline
\end{tabular}

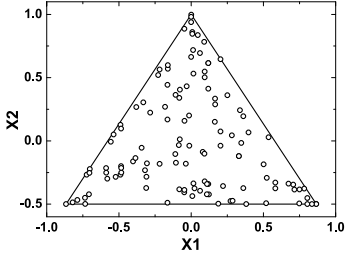

(a)

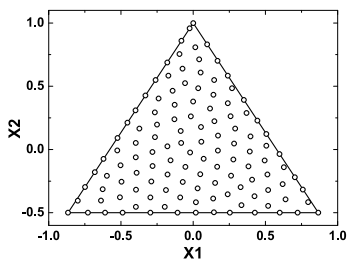

(f)

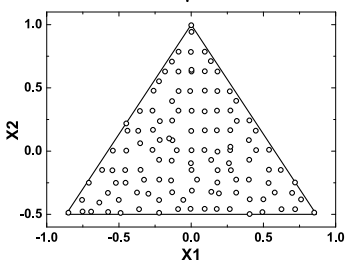

(k)

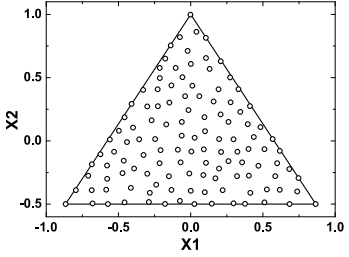

(b)

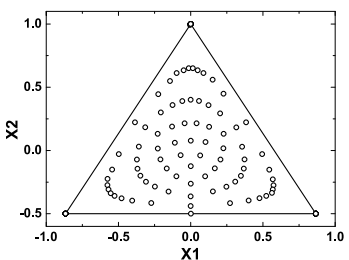

$(\mathrm{g})$

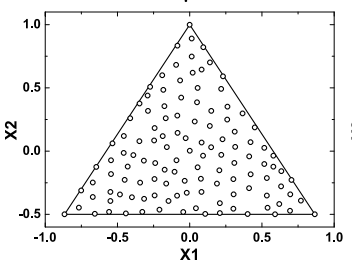

(1)

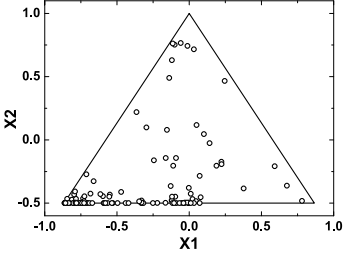

(c)

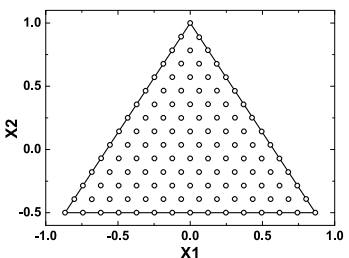

(h)

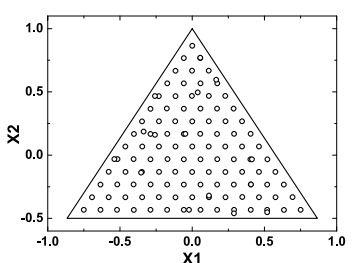

(m)

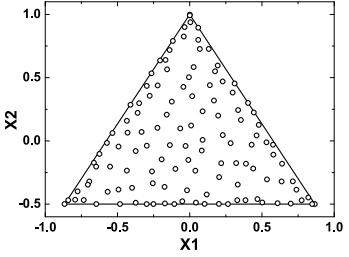

(d)

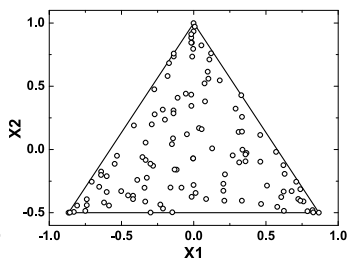

(i)

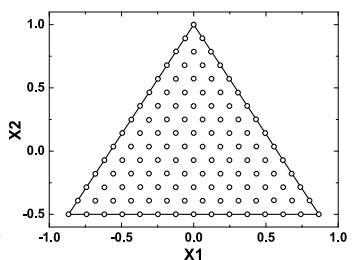

(n)

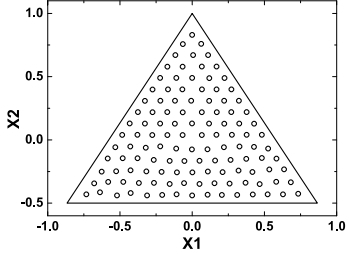

(e)

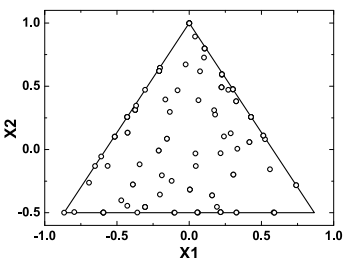

(j)

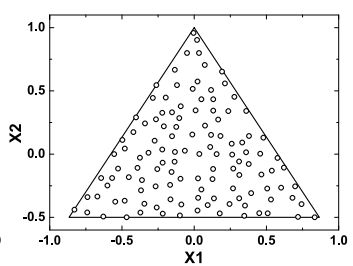

(o)

Fig. 5. Best solution set of the 15 algorithms on a tri-objective ML-DMP instance where the search space is precisely the optimal polygon, and its corresponding IGD result. The associated index of the algorithm represents the number of runs (out of all ten runs) in which the obtained solutions have a good coverage over the optimal polygon. (a) NSGA-II (10) IGD = 5.78E-2. (b) SPEA2 (10) IGD = 4.26E-2. (c) AR (0) IGD = 1.06E-1. (d) IBEA (10) $\mathrm{IGD}=4.76 \mathrm{E}-2$. (e) $\epsilon$-MOEA (10) IGD = 4.24E-2. (f) SMS-EMOA (10) IGD = 4.12E-2. (g) MOEA/D-TCH (10) IGD = 7.00E-2. (h) MOEA/D-PBI (10) IGD = 4.05E-2. (i) DMO (10) IGD = 5.65E-2. (j) HypE (10) IGD = 7.38E-2. (k) GrEA (10) IGD = 4.36E-2. (1) Two_Arch2 (10) IGD = 4.27E-2. (m) AGE-II (10) IGD = 4.25E-2. (n) NSGA-III (10) IGD = 4.05E-2. (o) SPEA2+SDE (10) IGD = 4.42E-2.

optimization subproblems by a set of weight vectors and an achievement scalarizing function, and then tackles them simultaneously. Here, two commonlyused achievement scalarizing functions, Tchebycheff and penalty-based boundary intersection, are considered in this paper (denoted as MOEA/D-TCH and MOEA/D-PBI).

8) Diversity Management Operator (DMO) [58]: DMO is an attempt of using a diversity management operator to adjust the diversity requirement in the selection process of evolutionary many-objective optimization. By comparing the boundary values between the current population and the Pareto front, the diversity maintenance mechanism is controlled (i.e., activated or inactivated).

9) Hypervolume Estimation Algorithm (HypE) [59]: As a representative indicator-based algorithm for manyobjective optimization, HypE adopts the Monte Carlo simulation to approximate the exact $\mathrm{HV}$ value, thereby significantly reducing the time cost of the $\mathrm{HV}$ calculation.

10) Grid-Based Evolutionary Algorithm (GrEA) [60]: GrEA explores the potential of the use of the grid in manyobjective optimization. In GrEA, a set of grid-based criteria are introduced to guide the search toward the optimal front, and a grid-based fitness adjustment strategy 


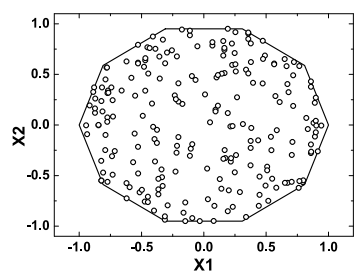

(a)

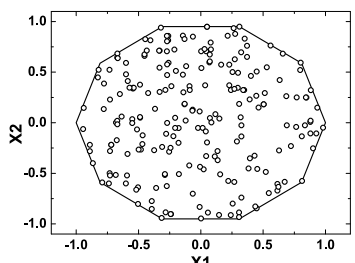

(f)

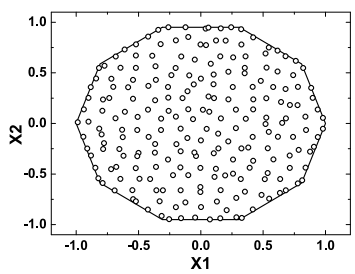

$(\mathrm{k})$

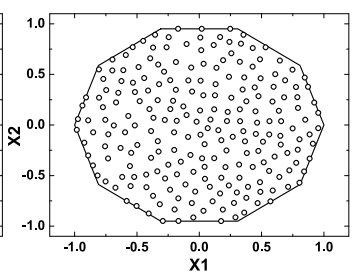

(b)

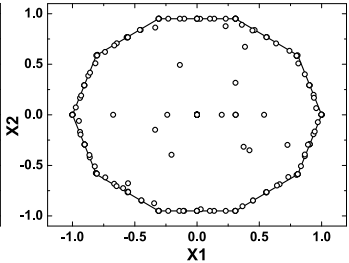

(g)

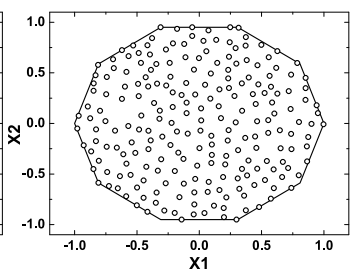

(l)

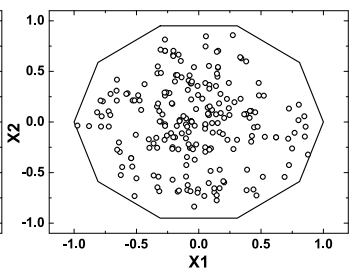

(c)

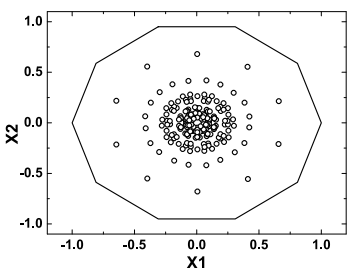

(h)

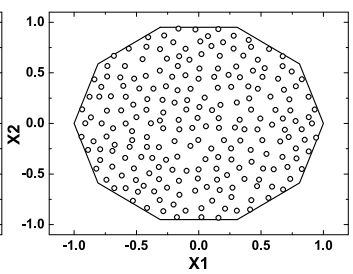

(m)

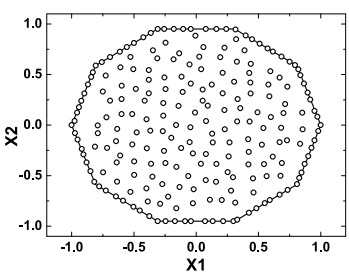

(d)

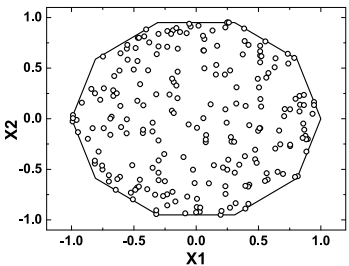

(i)

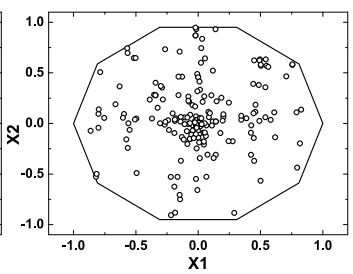

(n)

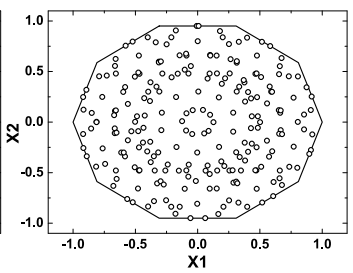

(e)

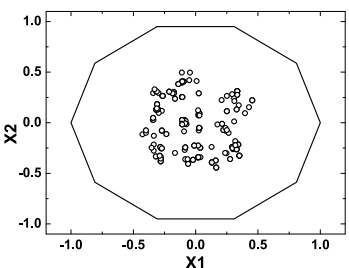

(j)

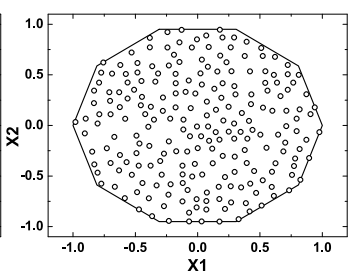

(o)

Fig. 6. Best solution set of the 15 algorithms on a ten-objective ML-DMP instance where the search space is precisely the optimal polygon, and its corresponding IGD result. The associated index of the algorithm represents the number of runs (out of all ten runs) in which the obtained solutions have a good coverage over the optimal polygon. (a) NSGA-II (10) IGD = 6.01E-2. (b) SPEA2 (10) IGD = 4.65E-2. (c) AR (9) IGD = 8.11E-2. (d) IBEA (10) IGD $=4.98 \mathrm{E}-2$. (e) $\epsilon$-MOEA (10) IGD $=5.40 \mathrm{E}-2$. (f) SMS-EMOA (10) IGD $=6.18 \mathrm{E}-2$. (g) MOEA/D-TCH (0) IGD = 1.30E-1. (h) MOEA/D-PBI (0)IGD = 1.51E-1. (i) DMO (10) IGD = 6.00E-2. (j) HypE (0) IGD = 2.62E-1. (k) GrEA (10) IGD = 4.87E-2. (1) Two_Arch2 (10) IGD = 4.78E-2. (m) AGE-II (10) IGD = 4.69E-2. (n) NSGA-III (0) IGD = 9.03E-2. (o) SPEA2+SDE (10) IGD = 4.84E-2.

TABLE II

Population Size, the $\epsilon$ Setting in $\epsilon$-MOEA, the Number of Reference Points/Directions $(h)$ Along EacH OBjective in the DeCOMPOSITION-BASED Algorithms MOEA/D-TCH, MOEA/D-PBI, AND NSGA-III

\begin{tabular}{l|c|c|c}
\hline Test Instance & $\epsilon$ & $h$ & Population Size \\
\hline Group I, 3-Obj. & 0.095 & 14 & 120 \\
Group II, 3-Obj & 0.085 & 14 & 120 \\
Group III, 3-Obj & 8.000 & 14 & 120 \\
Group II, 4-Obj & 0.120 & 7 & 120 \\
Group III, 4-Obj & 10.00 & 7 & 120 \\
Group II, 5-Obj & 0.135 & 5 & 128 \\
Group III, 5-Obj & 10.00 & 5 & 128 \\
Group I, 10-Obj & 0.179 & 3 & 220 \\
Group II, 10-Obj & 0.179 & 3 & 220 \\
Group III, 10-Obj & 10.00 & 3 & 220 \\
\hline
\end{tabular}

to maintain an extensive and uniform distribution among individuals.

11) Two-Archive Algorithm 2 (Two_Arch2) [61]: As a bi-population evolutionary algorithm, Two_Arch2 considers different selection criteria in the two archive sets, with one set being guided by the indicator $I_{\text {epsilon+ }}$ (from IBEA [54]) and the other by Pareto dominance and a $L_{p}$-norm-based distance measure, where $p$ is set to $1 / \mathrm{m}$.

12) Approximation-Guided Evolutionary Algorithm II (AGE-II) [62]: AGE-II incorporates a formal notion of approximation into an EMO algorithm. To improve the original AGE algorithm [66] suffering from heavy computational cost, AGE-II introduces an adaptive $\epsilon$-dominance approach to balance the convergence speed and runtime. Also, the mating selection strategy is redesigned to emphasize the population diversity.

13) NSGA-III [63]: NSGA-III is a recent many-objective algorithm whose framework is based on NSGA-II but with significant changes in the selection mechanism. Instead of the crowding distance, NSGA-III uses a decomposition-based niching technique to maintain diversity by a set of well-distributed weight vectors.

14) SPEA2 With Shift-Based Density Estimation $(S P E A 2+S D E)$ [46]: Shifting individuals' position before estimating their density, SDE can make Paretobased algorithms work effectively in many-objective optimization. In contrast to traditional density estimation which only involves individuals' distribution, SDE covers both distribution and convergence information of individuals. The Pareto-based algorithm SPEA2 has been demonstrated to be promising when working with SDE.

\section{General Experimental Setting}

A crossover probability $p_{c}=1.0$ and a mutation probability $p_{m}=1 / n$ (where $n$ denotes the number of decision variables) were used. The operators for crossover and mutation are simulated binary crossover and polynomial mutation with both distribution indexes 20. For newly-produced individuals which are located in the constrained areas of the ML-DMP, we simply reproduce them until they are feasible. 


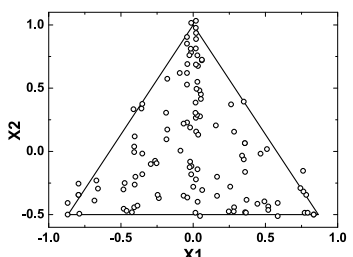

(a)

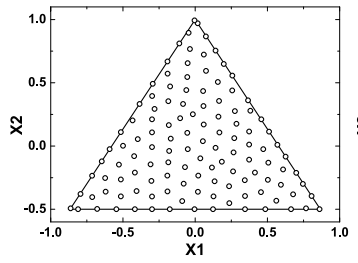

(f)

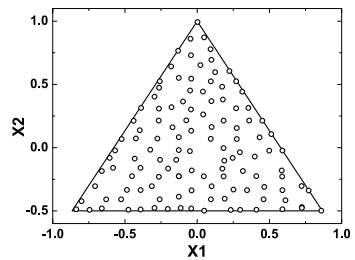

(k)

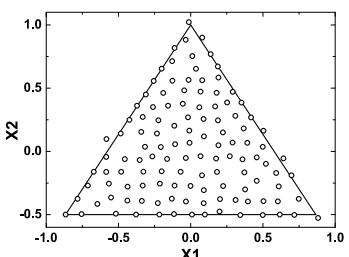

(b)

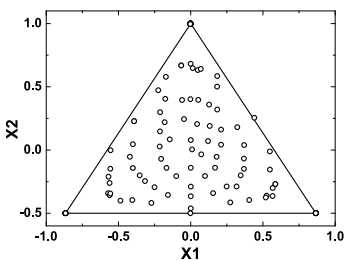

(g)

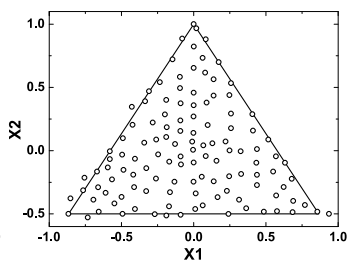

(1)

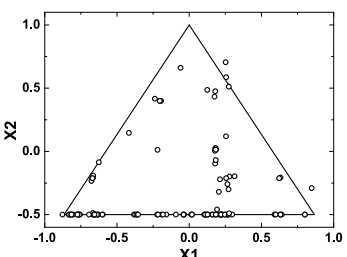

(c)

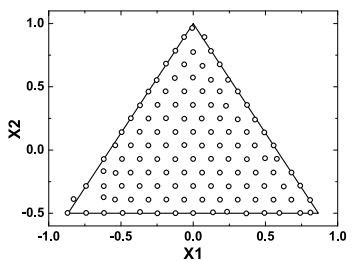

(h)

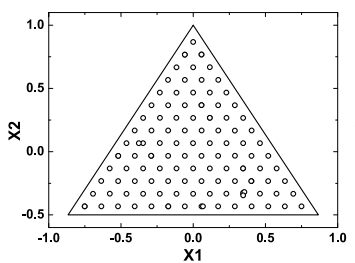

(m)

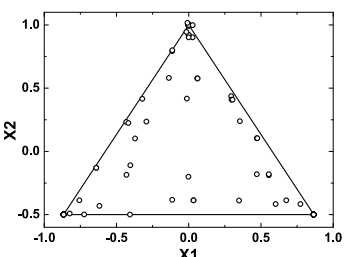

(d)

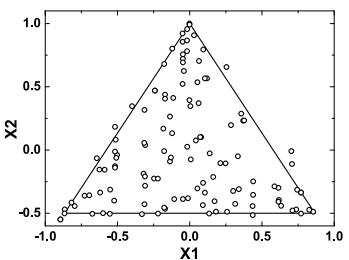

(i)

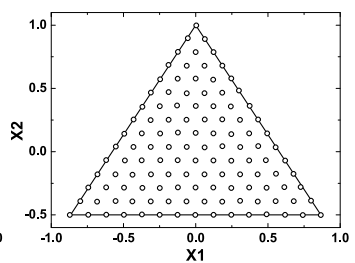

(n)

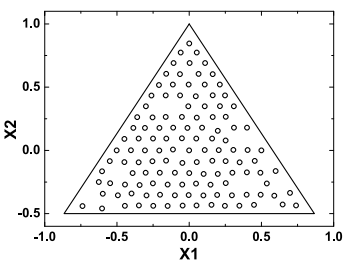

(e)

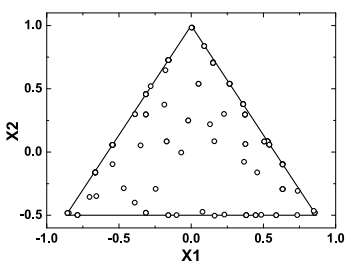

(j)

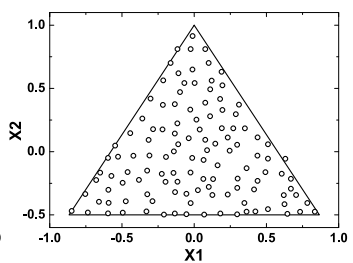

(o)

Fig. 7. Best solution set of the 15 algorithms on a tri-objective ML-DMP instance where the search space is [-100, 100 $]^{2}$, and its corresponding generational distance (GD) and IGD results. The associated indexes $\left(I_{1}, I_{2}\right)$ of the algorithm, respectively, represent the number of runs (out of all ten runs) in which all obtained solutions converge into (or are close to) the optimal polygon and the number of runs in which the obtained solutions have a good coverage over the optimal polygon. (a) NSGA-II $(10,10) \mathrm{GD}=3.10 \mathrm{E}-3$, IGD = 6.38E-2. (b) SPEA2 $(10,10) \mathrm{GD}=1.25 \mathrm{E}-3, \mathrm{IGD}=4.28 \mathrm{E}-2 .(\mathrm{c}) \mathrm{AR}(10,0) \mathrm{GD}=$ $2.65 \mathrm{E}-3, \mathrm{IGD}=1.16 \mathrm{E}-1$. (d) IBEA $(10,0) \mathrm{GD}=2.24 \mathrm{E}-4, \mathrm{IGD}=1.05 \mathrm{E}-1$. (e) $\epsilon-\mathrm{MOEA}(10,10) \mathrm{GD}=0.00 \mathrm{E}+0, \mathrm{IGD}=4.32 \mathrm{E}-2$. (f) SMS-EMOA $(10,10) \mathrm{GD}=2.98 \mathrm{E}-5, \mathrm{IGD}=4.16 \mathrm{E}-2 .(\mathrm{g}) \mathrm{MOEA} / \mathrm{D}-\mathrm{TCH}(10,10) \mathrm{GD}=2.51 \mathrm{E}-5, \mathrm{IGD}=6.45 \mathrm{E}-2$. (h) MOEA/D-PBI $(10,10) \mathrm{GD}=4.01 \mathrm{E}-5, \mathrm{IGD}$ $=4.17 \mathrm{E}-2$. (i) $\mathrm{DMO}(9,10) \mathrm{GD}=2.43 \mathrm{E}-3$, IGD = 6.17E-2. (j) HypE $(10,10) \mathrm{GD}=1.37 \mathrm{E}-4$, IGD = 8.09E-2. (k) GrEA (10, 7) GD = 5.07E-6, IGD $=4.53 \mathrm{E}-2$. (1) Two_Arch2 $(10,10) \mathrm{GD}=1.89 \mathrm{E}-3$, IGD = 4.64E-2. (m) AGE-II $(10,10)$ GD $=0.00 \mathrm{E}+0$, IGD $=4.27 \mathrm{E}-2$. (n) NSGA-III $(10,10)$ GD $=2.88 \mathrm{E}-4, \mathrm{IGD}=4.06 \mathrm{E}-2$. (o) $\operatorname{SPEA} 2+\mathrm{SDE}(10,10) \mathrm{GD}=1.96 \mathrm{E}-4, \mathrm{IGD}=4.37 \mathrm{E}-2$.

The termination criterion of the examined algorithms was 15000,30000 , and 60000 evaluations for types I-III of the ML-DMP instances, respectively. In the decomposition-based algorithms, the population size, which is determined by the number of reference points/directions $(h)$ along each objective, cannot be specified arbitrarily. In the experimental studies, we set $h$ to $14,7,5$, and 3 for the three-, four-, five-, and tenobjective ML-DMP, respectively. In addition, for some of the tested algorithms, such as NSGA-II and NSGA-III, the population size needs to be divisible by 4 . In view of these two requirements, we specify the population size (and the archive set) to $120,120,128$, and 220 for the three-, four-, five-, and ten-objective ML-DMPs. In $\epsilon$-MOEA, the size of the archive set is determined by parameter $\epsilon$. For a fair comparison, we set $\epsilon$ such that the archive set is approximately of the same size as that of the other algorithms. Table I summarizes parameter settings as well as the source of all the algorithms. The setting of these parameters in our experimental studies either follows the suggestion in their original papers or has been found to enable the algorithm to perform better on the ML-DMP.

\section{EXPERIMENTAL Results}

In this section, we examine the search behavior of the 15 EMO algorithms by demonstrating their solution sets in the 2-D decision space for the three types of ML-DMP instances described in the previous section. Each algorithm was executed ten independent runs, from which we displayed the best solution set (determined by the IGD indicator [28]) of one run. For a quantitative understanding, the GD [67] and IGD results of the best solution set were also included in the figures. GD and IGD are two popular performance indicators which assess a solution set's convergence and comprehensive performance (i.e., both convergence and diversity), respectively. To calculate GD, we considered the average Euclidean distance of the solutions to the optimal polygon. That is, if a solution is inside the optimal polygon the distance is zero; otherwise, it is the distance from the solution to its closest edge of the polygon. For IGD, we randomly generated 50000 points inside the optimal polygon and then calculated the average Euclidean distance from these points to their closest solution in the considered solution set.

In addition, to examine the stability of the algorithms in terms of convergence and diversity individually, we provide two number indexes $I_{1}$ and $I_{2}$, with $I_{1}$ being the number of runs (out of all ten runs) in which the final solutions obtained by the tested algorithm converge into (or are very close to) the optimal polygon and $I_{2}$ being the number of runs in which the solutions have a good coverage over the optimal polygon. These two indexes are determined by GD and IGD, respectively.

\section{A. Type I ML-DMP}

Fig. 5 shows the best one-run solution sets obtained by the 15 algorithms on the tri-objective type I ML-DMP instance 


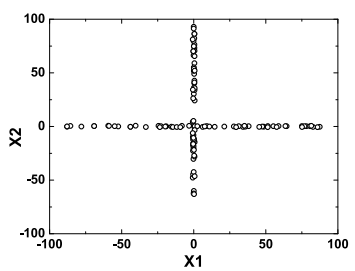

(a)

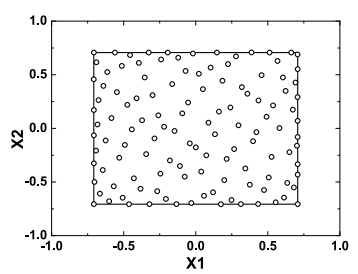

(f)

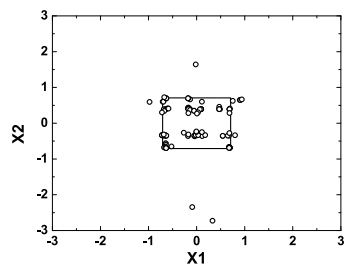

(k)

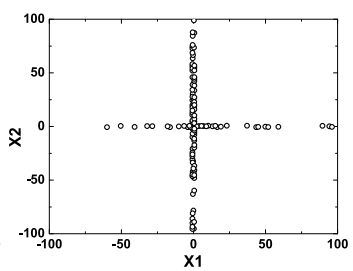

(b)

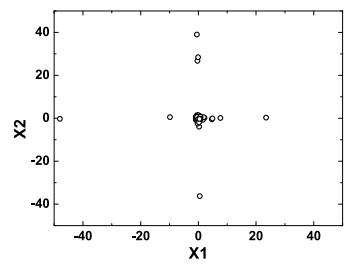

(g)

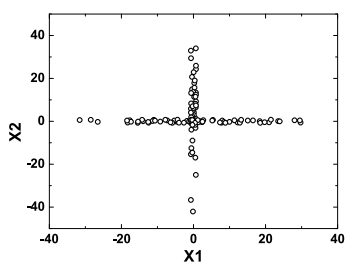

(l)

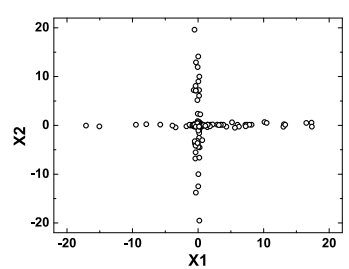

(c)

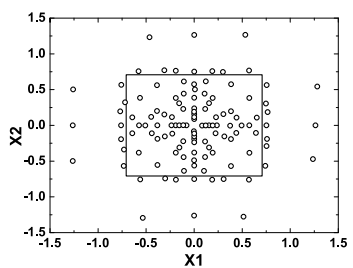

(h)

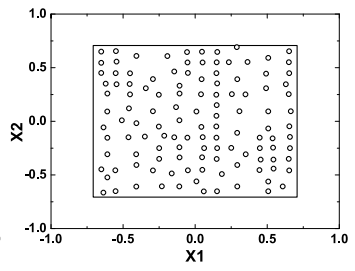

(m)

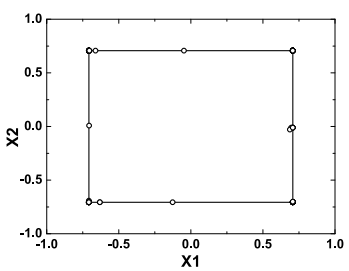

(d)

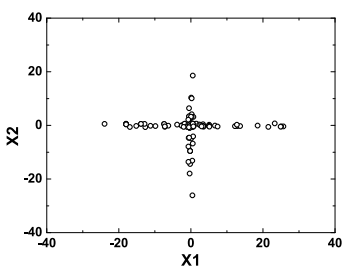

(i)

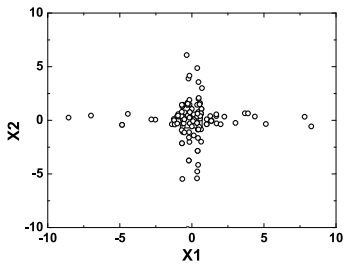

(n)

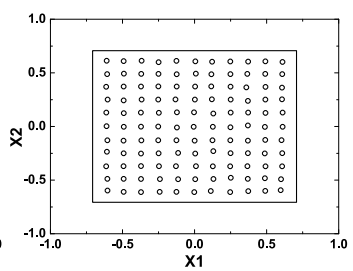

(e)

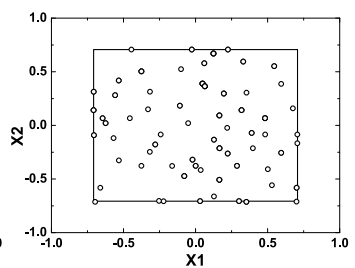

(j)

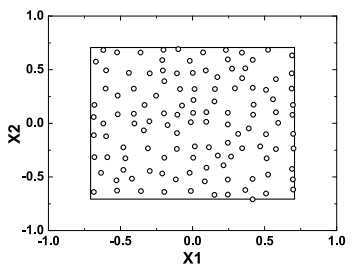

(o)

Fig. 8. Best solution set of the 15 algorithms on a four-objective ML-DMP instance where the search space is [-100, 100] ${ }^{2}$, and its corresponding GD and IGD results. The associated indexes $\left(I_{1}, I_{2}\right)$ of the algorithm, respectively, represent the number of runs (out of all ten runs) in which all obtained solutions converge into (or are close to) the optimal polygon and the number of runs in which the obtained solutions have a good coverage over the optimal polygon. (a) NSGA-II $(0,0) \mathrm{GD}=4.20 \mathrm{E}+1$, IGD = 5.45E-1. (b) SPEA2 (0, 0) GD = 3.63E+1, IGD = 4.92E-1. (c) AR (0, 0) GD = 3.81E+0, IGD = 1.42E-1. (d) $\operatorname{IBEA}(10,0) \mathrm{GD}=1.70 \mathrm{E}-4$, IGD = 2.72E-1. (e) $\epsilon$-MOEA $(10,10) \mathrm{GD}=0.00 \mathrm{E}+0$, IGD $=5.32 \mathrm{E}-2$. (f) SMS-EMOA $(10,10)$ GD $=3.21 \mathrm{E}-5$, $\mathrm{IGD}=5.78 \mathrm{E}-2 .(\mathrm{g}) \mathrm{MOEA} / \mathrm{D}-\mathrm{TCH}(0,0) \mathrm{GD}=8.03 \mathrm{E}-1, \mathrm{IGD}=9.63 \mathrm{E}-2 .(\mathrm{h}) \mathrm{MOEA} / \mathrm{D}-\mathrm{PBI}(0,10) \mathrm{GD}=6.71 \mathrm{E}-2, \mathrm{IGD}=7.66 \mathrm{E}-2 .(\mathrm{i}) \mathrm{DMO}(0,0)$ $\mathrm{GD}=7.60 \mathrm{E}+0, \mathrm{IGD}=1.50 \mathrm{E}-1$. (j) $\operatorname{HypE}(10,10) \mathrm{GD}=2.83 \mathrm{E}-4, \mathrm{IGD}=8.44 \mathrm{E}-2$. (k) GrEA $(5,0)$ GD $=2.72 \mathrm{E}-2, \mathrm{IGD}=1.56 \mathrm{E}-1$. (1) Two_Arch2 $(0,0) \mathrm{GD}=1.53 \mathrm{E}+1, \mathrm{IGD}=2.11 \mathrm{E}-1 .(\mathrm{m})$ AGE-II $(10,10) \mathrm{GD}=0.00 \mathrm{E}+0, \mathrm{IGD}=5.36 \mathrm{E}-2 .(\mathrm{n}) \mathrm{NSGA}-\mathrm{III}(0,0)$ GD $=4.88 \mathrm{E}+0, \mathrm{IGD}=1.23 \mathrm{E}-1$. (o) $\operatorname{SPEA} 2+\operatorname{SDE}(10,10) \mathrm{GD}=0.00 \mathrm{E}+0, \mathrm{IGD}=5.34 \mathrm{E}-2$.

where the search space is precisely the optimal triangle. This allows an independent examination of the algorithms' performance in maintaining diversity. As can be seen in the figure, the solutions of all the algorithms except AR are widely distributed over the triangle, which verifies their ability in diversifying the population on the tri-objective problem. Among these algorithms, however, some fail to maintain the uniformity of distribution, leading to the solutions crowded (or even overlapping) in some areas but sparse in some others. Such algorithms includes NSGA-II, DMO, HypE, and MOEA/D-TCH; the last one, interestingly, has a regularly-distributed solution set. In contrast, the solutions obtained by $\epsilon$-MOEA and AGE-II have an excellent uniformity, but cannot cover the boundary of the triangle. SPEA2, IBEA, GrEA, Two_Arch2, and SPEA2+SDE are the algorithms which achieve a good balance between uniformity and extensity. In addition, three well-known algorithms, SMSEMOA, MOEA/D-PBI, and NSGA-III, tend to have a perfect performance on this problem, with their solutions being highly uniform over the whole triangle.

The above observations show that most of the tested EMO algorithms are able to effectively maintain solutions' diversity on the tri-objective instance. So, how do they perform when more objectives are involved? Fig. 6 gives the results of the best solution sets of the 15 algorithms on the ten-objective

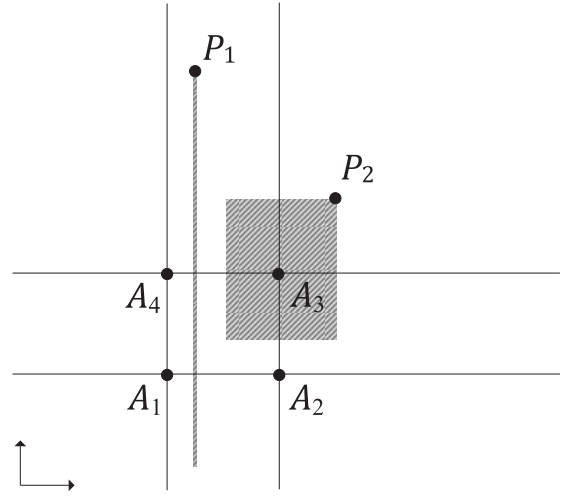

Fig. 9. Illustration of the difficulty for EMO algorithms to converge on the four-objective ML-DMP problem. where $A_{1}-A_{4}$ are the four vertexes of the optimal polygon. The shadows are the regions that dominate $P_{1}$ and $P_{2}$, respectively.

type I instance. We here do not show the results on fourand five-objective instances since the algorithms perform very similarly on all the type I instances with more than three objectives. As shown in the figure, most of the algorithms have the similar pattern as in the tri-objective instance. This means that their ability of maintaining diversity does not degrade with the increase of the number of objectives. That is, if there are 


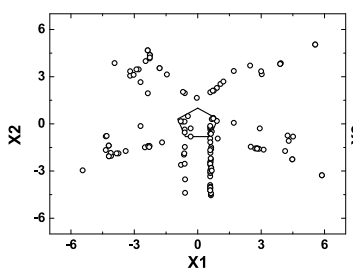

(a)

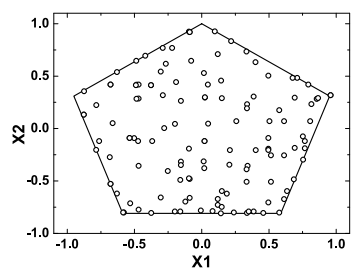

(f)

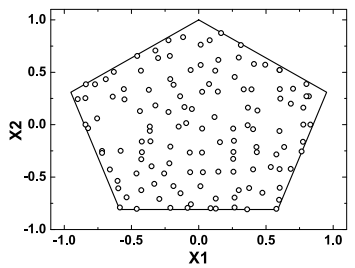

(k)

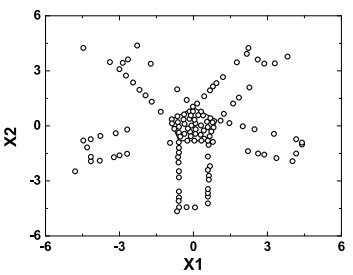

(b)

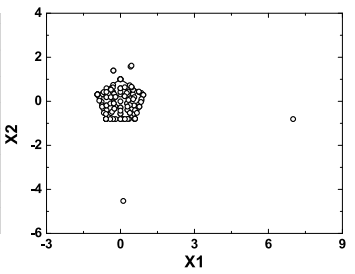

$(\mathrm{g})$

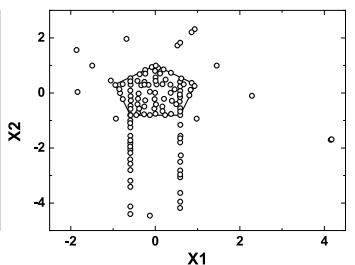

(1)

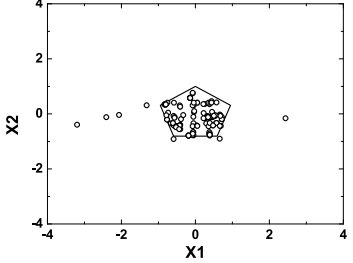

(c)

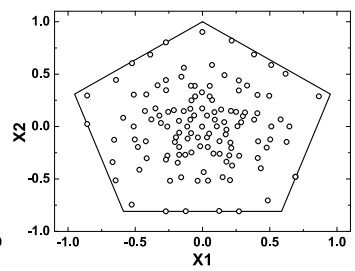

(h)

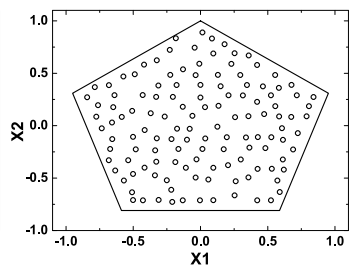

(m)

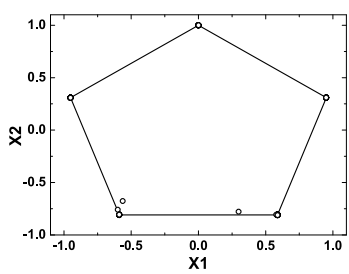

(d)

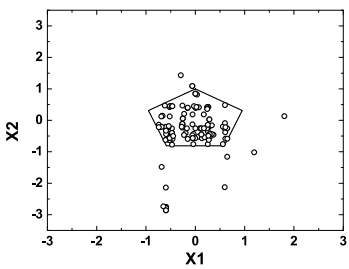

(i)

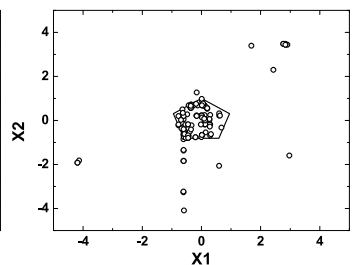

(n)

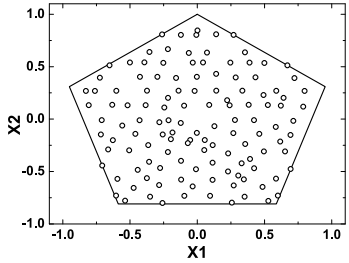

(e)

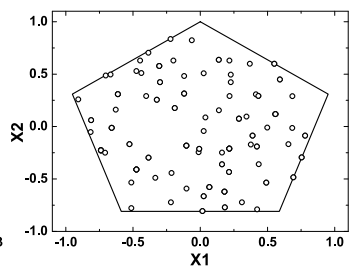

(j)

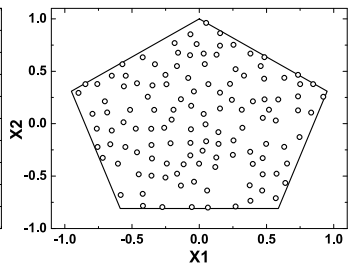

(o)

Fig. 10. Best solution set of the 15 algorithms on a five-objective ML-DMP instance where the search space is [-100, 100] ${ }^{2}$, and its corresponding GD and IGD results. The associated indexes $\left(I_{1}, I_{2}\right)$ of the algorithm, respectively, represent the number of runs (out of all ten runs) in which all obtained solutions converge into (or are close to) the optimal polygon and the number of runs (out of all ten runs) in which the obtained solutions have a good coverage over the optimal polygon. (a) NSGA-II $(0,0) \mathrm{GD}=2.48 \mathrm{E}+0$, IGD $=2.40 \mathrm{E}-1$. (b) SPEA2 $(0,0) \mathrm{GD}=1.59 \mathrm{E}+0$, IGD $=1.01 \mathrm{E}-1$. (c) AR (0, 0) GD $=1.16 \mathrm{E}-1$, $\mathrm{IGD}=1.12 \mathrm{E}-1$. (d) IBEA $(10,0) \mathrm{GD}=2.73 \mathrm{E}-4$, IGD $=4.36 \mathrm{E}-1$. (e) $\epsilon$-MOEA $(10,10) \mathrm{GD}=0.00 \mathrm{E}+0$, IGD $=5.78 \mathrm{E}-2$. (f) SMS-EMOA (10, 10) GD $=1.81 \mathrm{E}-4$, IGD $=6.97 \mathrm{E}-2 .(\mathrm{g}) \mathrm{MOEA} / \mathrm{D}-\mathrm{TCH}(0,10) \mathrm{GD}=2.50 \mathrm{E}-1, \mathrm{IGD}=7.76 \mathrm{E}-2$. (h) MOEA/D-PBI $(10,10)$ GD $=0.00 \mathrm{E}+0, \mathrm{IGD}=7.33 \mathrm{E}-2$. (i) $\mathrm{DMO}(0,0) \mathrm{GD}=2.76 \mathrm{E}-1$, IGD $=1.15 \mathrm{E}-1$. (j) $\mathrm{HypE}(10,10) \mathrm{GD}=0.00 \mathrm{E}+0$, IGD $=8.57 \mathrm{E}-2$. (k) GrEA $(9,9) \mathrm{GD}=0.00 \mathrm{E}+0$, IGD $=6.53 \mathrm{E}-2$. (1) Two_Arch2 $(0,7) \mathrm{GD}=6.30 \mathrm{E}-1$, IGD $=7.77 \mathrm{E}-2$. (m) AGE-II $(10,10)$ GD $=0.00 \mathrm{E}+0$, IGD $=5.77 \mathrm{E}-2$. (n) NSGA-III $(0,0)$ GD $=6.23 \mathrm{E}-1$, IGD $=1.08 \mathrm{E}-1$. (o) SPEA2 $+\mathrm{SDE}(9,10) \mathrm{GD}=0.00 \mathrm{E}+0, \mathrm{IGD}=5.80 \mathrm{E}-2$.

sufficient well-converged solutions being produced during the evolutionary process, these algorithms can diversify them well even in the high-dimensional space.

Nevertheless, there do exist some algorithms which scale up badly with the number of objectives. They include $\epsilon$-MOEA, SMS-EMOA, MOEA/D-TCH, MOEA/D-PBI, HypE, and NSGA-III. It is worth mentioning that all of these algorithms do not use directly density-based methods in diversity maintenance. $\epsilon$-MOEA maintains the population diversity by the $\epsilon$ dominance, SMS-EMOA and HypE rely on the HV indicator, and MOEA/D-TCH, MOEA/D-PBI, and NSGA-III use the decomposition-based strategy. The failure of $\epsilon$-MOEA in obtaining a uniformly-distributed solution set suggests the difficulty that the $\epsilon$ dominance faces in the high-dimensional space. One possible explanation of SMS-EMOA and HypE's underperformance on the ten-objective instance is that an approximate estimation of the HV contribution may affect the performance of the algorithms. In addition, it is not surprising that the three decomposition-based algorithms cannot maintain solutions' diversity on this instance since the ML-DMP with more than three objectives has a degenerate Pareto front (i.e., the dimensionality of the Pareto front is less than the number of objectives), on which decomposition-based algorithms commonly struggle [31], [63]. Finally, an interesting observation is that AR which does not use any diversity maintenance scheme during the evolutionary process performs better than some of the other algorithms (such as MOEA/D-TCH and HypE). This indicates that random selection could even pick out more diversified individuals than some decomposition-based or indicator-based selection in high-dimensional ML-DMP problems.

\section{B. Type II ML-DMP}

The search space of the type II ML-DMP problem is $[-100,100]^{2}$, significantly larger than the optimal region $\left(<[-1,1]^{2}\right)$, thus providing a challenge for EMO algorithms to achieve a balance between convergence and diversity. Fig. 7 shows the best one-run solution sets obtained by the 15 algorithms on the tri-objective instance. As shown, all the algorithms have a good convergence, with their individuals inside (or very close to) the optimal triangle. Also, the solution sets obtained by most algorithms are distributed similarly as on the type I ML-DMP. One exception is IBEA, which performs significantly worse than on the type I instance since many of its solutions are overlapping. This indicates that the measure of IBEA's indicator prefers overlapping solutions to poorly-converged ones.

The above results show the ability of the examined algorithms in balancing convergence and diversity on the 


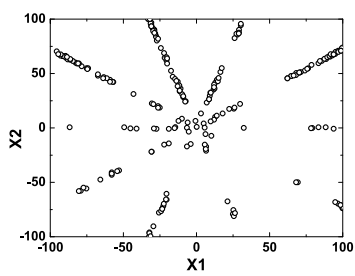

(a)

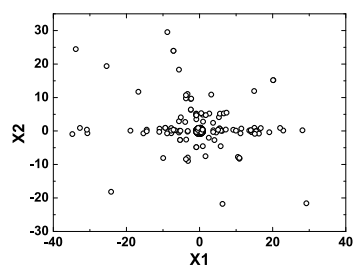

(f)

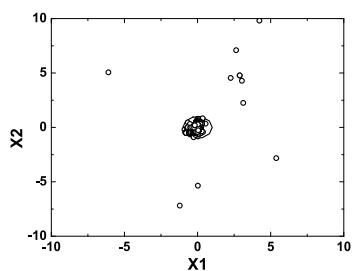

(k)

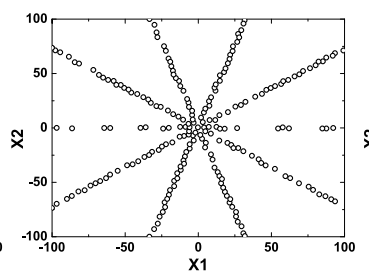

(b)

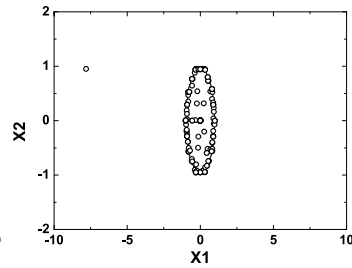

(g)

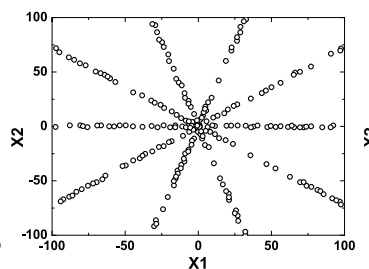

(1)

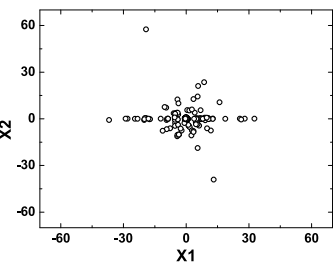

(c)

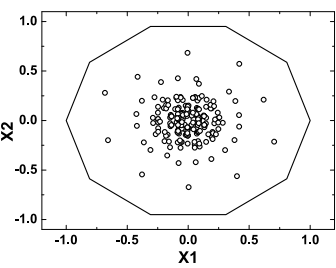

(h)

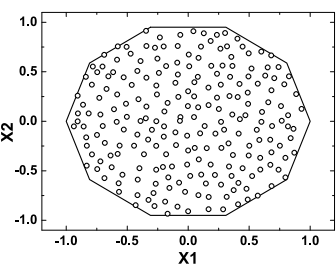

(m)

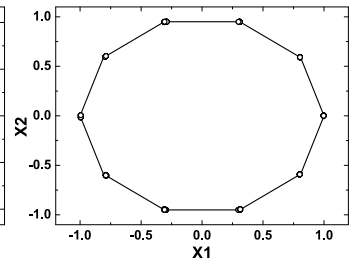

(d)

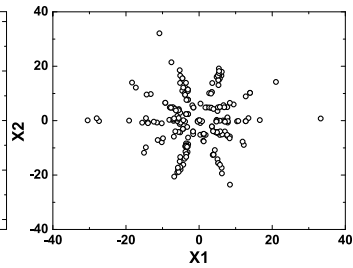

(i)

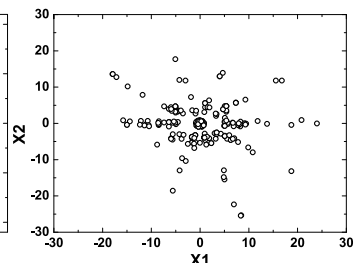

(n)

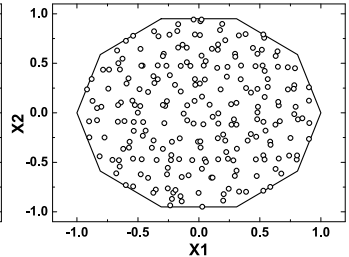

(e)

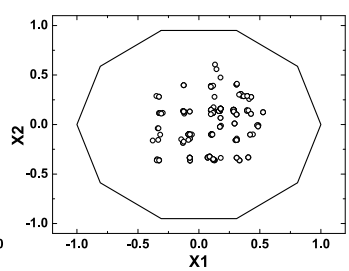

(j)

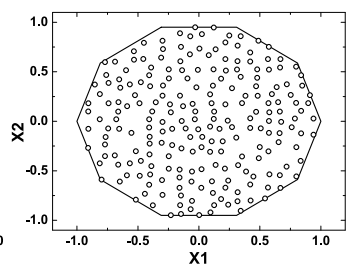

(o)

Fig. 11. Best solution set of the 15 algorithms on a ten-objective ML-DMP instance where the search space is [-100, 100] ${ }^{2}$, and its corresponding GD and IGD results. The associated indexes $\left(I_{1}, I_{2}\right)$ of the algorithm, respectively, represent the number of runs (out of all ten runs) in which all obtained solutions converge into (or are close to) the optimal polygon and the number of runs in which the obtained solutions have a good coverage over the optimal polygon. (a) NSGA-II $(0,0) \mathrm{GD}=7.03 \mathrm{E}+1$, IGD $=1.11 \mathrm{E}+0$. (b) SPEA2 $(0,0) \mathrm{GD}=5.62 \mathrm{E}+1$, IGD $=8.44 \mathrm{E}-1$. (c) AR $(0,0)$ GD $=6.21 \mathrm{E}+0, \mathrm{IGD}=1.19 \mathrm{E}-1$. (d) $\operatorname{IBEA}(10,0) \mathrm{GD}=0.00 \mathrm{E}+0, \mathrm{IGD}=3.70 \mathrm{E}-1$. (e) $\epsilon$-MOEA $(10,10) \mathrm{GD}=0.00 \mathrm{E}+0$, IGD $=5.31 \mathrm{E}-2$. (f) SMS-EMOA $(0,0) \mathrm{GD}=5.75 \mathrm{E}+0, \mathrm{IGD}$ $=1.02 \mathrm{E}-1$. (g) MOEA/D-TCH $(0,0) \mathrm{GD}=1.86 \mathrm{E}-1$, IGD $=1.39 \mathrm{E}-1$. (h) MOEA/D-PBI $(10,0) \mathrm{GD}=0.00 \mathrm{E}+0, \mathrm{IGD}=1.54 \mathrm{E}-1$. (i) DMO $(0,0)$ $\mathrm{GD}=1.05 \mathrm{E}+1, \mathrm{IGD}=4.97 \mathrm{E}-1$. (j) $\mathrm{HypE}(10,0) \mathrm{GD}=0.00 \mathrm{E}+0$, IGD $=2.56 \mathrm{E}-1$. (k) GrEA $(0,0) \mathrm{GD}=2.35 \mathrm{E}+0, \mathrm{IGD}=1.44 \mathrm{E}-1$. (1) Two_Arch2 $(0,0) \mathrm{GD}=5.24 \mathrm{E}+1, \mathrm{IGD}=5.06 \mathrm{E}-1 .(\mathrm{m})$ AGE-II $(10,10) \mathrm{GD}=0.00 \mathrm{E}+0, \mathrm{IGD}=4.76 \mathrm{E}-2 .(\mathrm{n}) \mathrm{NSGA}-\mathrm{III}(0,0)$ GD $=6.30 \mathrm{E}+0, \mathrm{IGD}=1.55 \mathrm{E}-1$. (o) $\operatorname{SPEA} 2+\mathrm{SDE}(10,10) \mathrm{GD}=0.00 \mathrm{E}+0, \mathrm{IGD}=4.79 \mathrm{E}-2$.

tri-objective ML-DMP. Then, how do they perform on the problem with more objectives? Fig. 8 shows the solution sets obtained by the 15 algorithms on the four-objective type II ML-DMP. As shown, only five algorithms, $\epsilon$-MOEA, SMSEMOA, AGE-II, SPEA2+SDE, and HypE, perform well on this problem, from which $\epsilon$-MOEA has an excellent uniformity and SMS-EMOA, AGE-II and SPEA2+SDE have a good balance between uniformity and extensity. Most of the remaining algorithms are unable to guide their population to converge into the optimal rectangle, with their solution sets typically distributed in the form of a cross.

Fig. 9 gives an illustration to explain why this happens. $P_{1}$ and $P_{2}$ are two solutions for a four-objective ML-DMP problem with four vertexes $A_{1}-A_{4} . P_{1}$ resides between two parallel target lines $\overleftrightarrow{A_{1} A_{4}}$ and $\overleftrightarrow{A_{2} A_{3}}$, and $P_{2}$ in the right upper area to the optimal square. As seen, the region that Pareto dominates $P_{1}$ is a line segment, far smaller than that dominating $P_{2}$, although $P_{1}$ is farther to the optimal polygon than $P_{2}$. In fact, any solution (outside the optimal polygon) located between a pair of parallel target lines is dominated by only a line segment parallel to these two lines; an improvement of its distance to the one line will lead to the degradation to the other. This property poses a big challenge not only for the algorithms who use Pareto dominance as the main selection criterion, such as NSGA-II, SPEA2, DMO, Two_Arch2 and NSGA-III, but also for some other modern algorithms, such as MOEA/D-TCH and GrEA. The solutions obtained by these algorithms can easily be distributed crisscross in the space.

Fig. 10 shows the solution sets obtained by the 15 algorithms on the five-objective instance. Similar to the situation on the four-objective instance, the Pareto-based EMO algorithms struggle to converge. This is because solutions in some regions (i.e., the boundary of the constrained polygons) are only dominated by one point in the pentagon. One difference from the four-objective situation is that all the solutions obtained by MOEA/D-PBI and GrEA can converge into the optimal region. This indicates that the difficulty of the MLDMP problem does not certainly increase with the number of objectives.

When the considered objective dimensionality of the MLDMP is 10, both parallel target lines and constrained areas are involved in the problem. This naturally leads to bigger challenges for EMO algorithms to balance the convergence and diversity. As can be seen in Fig. 11, only three algorithms, $\epsilon$-MOEA, AGE-II, and SPEA2+SDE, work well on the tenobjective instance. The solution sets of IBEA, MOEA/D-PBI, and HypE can converge into the optimal region but fail to cover the whole polygon. 


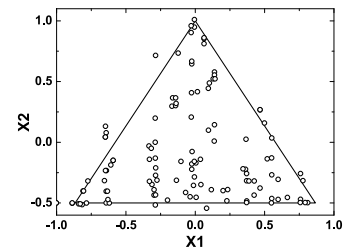

(a)

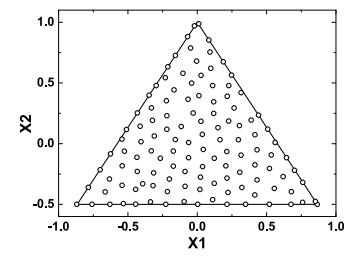

(f)

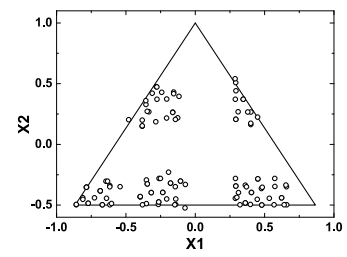

(k)

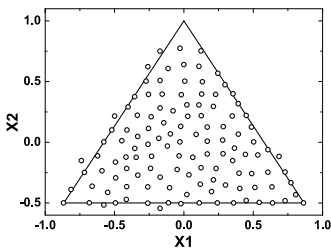

(b)

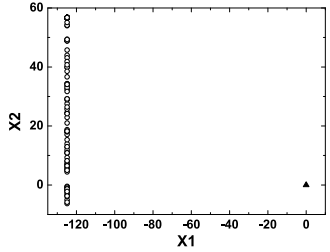

(g)

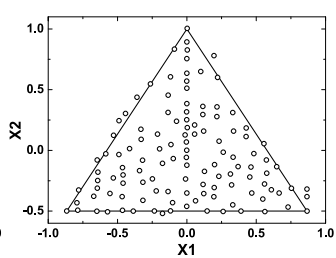

(1)

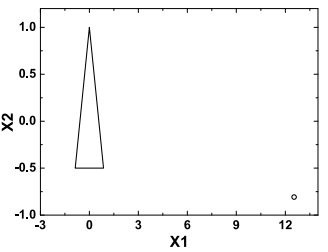

(c)

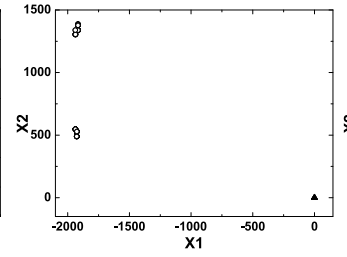

(h)

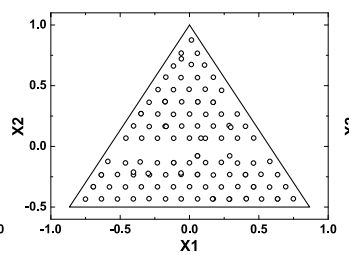

(m)

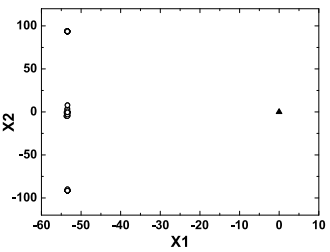

(d)

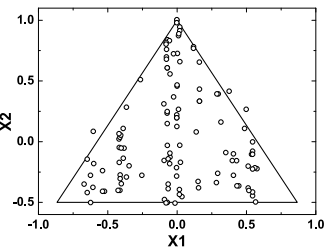

(i)

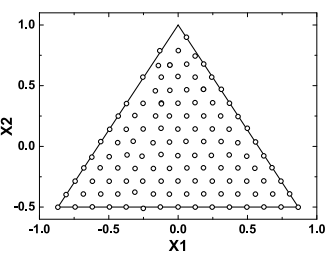

(n)

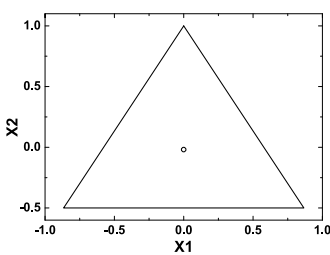

(e)

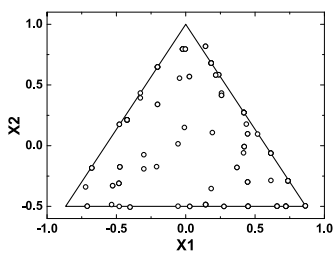

(j)

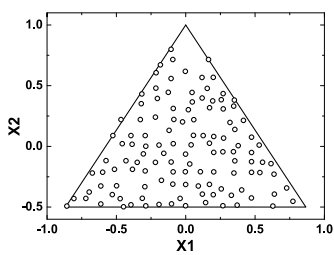

(o)

Fig. 12. Best solution set of the 15 algorithms on a tri-objective ML-DMP instance where the search space is $\left[-10^{10}, 10^{10}\right]^{2}$, and its corresponding GD and IGD results. The associated indexes $\left(I_{1}, I_{2}\right)$ of the algorithm, respectively, represent the number of runs (out of all ten runs) in which all obtained solutions converge into (or are close to) the optimal polygon and the number of runs in which the obtained solutions have a good coverage over the optimal polygon. (a) NSGA-II $(2,4) \mathrm{GD}=6.12 \mathrm{E}-3$, IGD $=7.08 \mathrm{E}-2$. (b) $\mathrm{SPEA} 2(2,3) \mathrm{GD}=1.46 \mathrm{E}-3$, IGD $=4.36 \mathrm{E}-2$. (c) $\mathrm{AR}(0,0) \mathrm{GD}=4.31 \mathrm{E}+1$, IGD $=4.72 \mathrm{E}+2$. (d) $\operatorname{IBEA}(0,0) \mathrm{GD}=2.18 \mathrm{E}+1, \mathrm{IGD}=1.31 \mathrm{E}+2$. (e) $\epsilon$-MOEA $(7,0) \mathrm{GD}=0.00 \mathrm{E}+0$, IGD $=5.06 \mathrm{E}-1$. (f) $\mathrm{SMS}-\mathrm{EMOA}(3,3) \mathrm{GD}=8.15 \mathrm{E}-5, \mathrm{IGD}=$ 4.31E-2. (g) MOEA/D-TCH $(0,0) \mathrm{GD}=8.40 \mathrm{E}+1$, IGD $=2.99 \mathrm{E}+2$. (h) MOEA/D-PBI $(0,0) \mathrm{GD}=7.19 \mathrm{E}+2, \mathrm{IGD}=1.99 \mathrm{E}+3$. (i) $\mathrm{DMO}(5,2) \mathrm{GD}=$ 4.01E-4, IGD = 7.60E-2. (j) $\operatorname{HypE}(4,2) \mathrm{GD}=7.19 \mathrm{E}-4$, IGD = 8.75E-2. (k) GrEA (4, 0) GD = 3.61E-4, IGD = 1.60E-1. (1) Two_Arch2 (5, 5) GD $=3.50 \mathrm{E}-3, \mathrm{IGD}=5.05 \mathrm{E}-2 .(\mathrm{m}) \mathrm{AGE}-\mathrm{II}(4,1) \mathrm{GD}=0.00 \mathrm{E}+0, \mathrm{IGD}=5.36 \mathrm{E}-2 .(\mathrm{n}) \mathrm{NSGA}-\mathrm{III}(4,2) \mathrm{GD}=3.70 \mathrm{E}-4, \mathrm{IGD}=4.25 \mathrm{E}-2 .(\mathrm{o}) \mathrm{SPEA} 2+\mathrm{SDE}$ $(8,5) \mathrm{GD}=2.33 \mathrm{E}-4, \mathrm{IGD}=4.98 \mathrm{E}-2$.

\section{Type III ML-DMP}

Type III ML-DMP hugely extends the problem's search space $\left(\left[-10^{10}, 10^{10}\right]\right)$ to test the algorithms' ability of leading solutions to converge toward the Pareto optimal region. Fig. 12 shows the best one-run solution sets obtained by the 15 algorithms on the tri-objective instance. An interesting observation is that different decomposition-based and indicator-based algorithms behave rather differently, such as IBEA versus SMS-EMOA and HypE, and MOEA/D versus NSGA-III. One explanation for this is that the Pareto dominance criterion can effectively guide the population into the optimal regionthe decomposition-based and indicator-based algorithms which use Pareto dominance as the primary selection criterion (i.e., SMS-EMOA, HypE, and NSGA-III) perform much better than those not using the Pareto dominance criterion (i.e., IBEA, MOEA/D-TCH, and MOEA/D-PBI). This has also been proven by the fact that some classic Pareto-based algorithms work well on this problem, such as NSGA-II and SPEA2. In addition, note that only one solution is obtained by $\epsilon$-MOEA. In fact, no matter how the $\epsilon$ value of the algorithm is set, there is always a sole solution left in the final archive set when the problem's search space becomes huge. This applies to all the type III ML-DMP instances with any number of objectives. Finally, it is worth mentioning that there is none of the tested algorithms able to obtain a stable performance in terms of both convergence and diversity, as shown by the two indexes $I_{1}$ and $I_{2}$ in the figure. This indicates that the proposed problem poses great challenges for EMO algorithms even in the 3-D space.

Consider the four- and five-objective instances shown in Figs. 13 and 14, respectively. Only AGE-II and SPEA2+SDE are able to find a well-converged, well-distributed solution set on both instances. HypE performs fairly well on the fourobjective instance, and SMS-EMOA occasionally converges for the five-objective instance. Interesting observations regarding the five-objective results are from SPEA2 and Two_Arch2 which sometimes have a good coverage over the optimal pentagon, but cannot lead all of their solutions into the optimal region.

The type III ten-objective ML-DMP is the hardest problem that we tested in this experimental study. As can be seen in Fig. 15, only SPEA2+SDE can obtain a good convergence and diversity on nearly half of the ten runs. Among the other algorithms, IBEA and $\epsilon$-MOEA can occasionally converge, but their solutions concentrate in either several boundary points or the central point of the polygon.

\section{Summary}

On the basis of the investigation on the three types of ten ML-DMP instances, the following observations of the $15 \mathrm{EMO}$ algorithms can be made.

1) Despite failing on the ML-DMP with two parallel target lines, the conventional Pareto-based algorithms NSGA-II and SPEA2 have shown their advantage on 


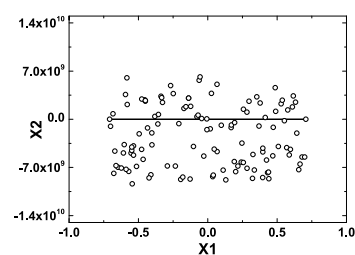

(a)

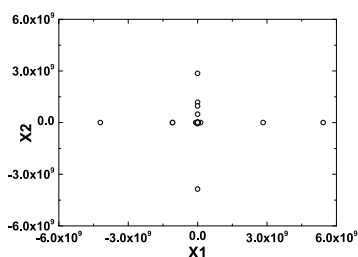

(f)

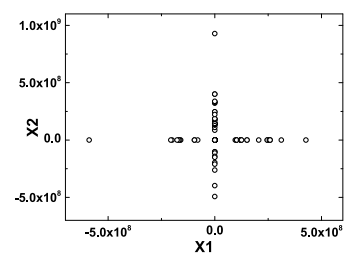

(k)

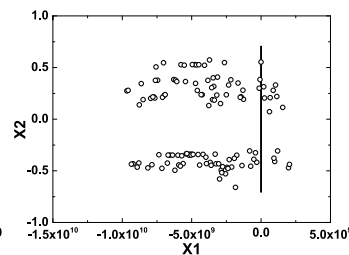

(b)

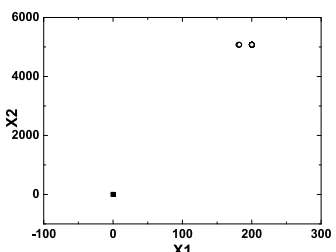

(g)

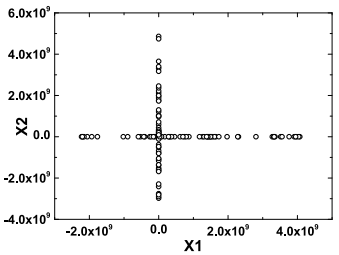

(1)

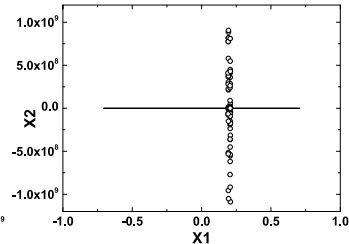

(c)

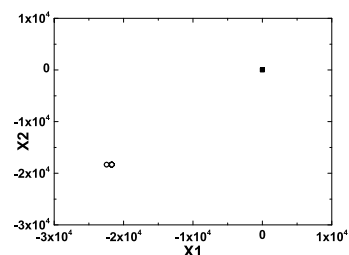

(h)

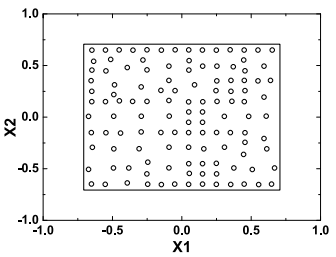

(m)

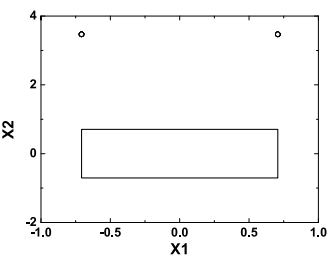

(d)

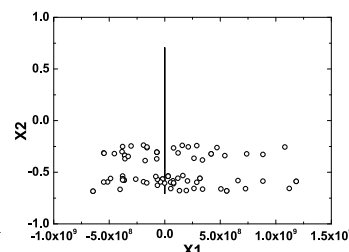

(i)

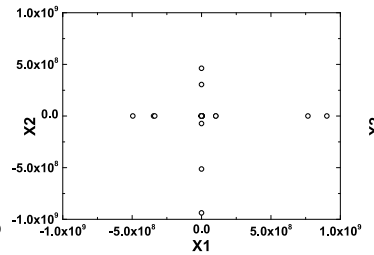

(n)

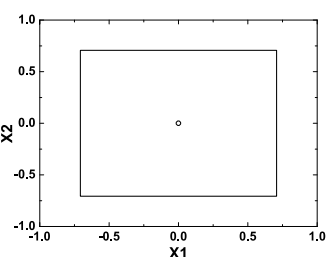

(e)

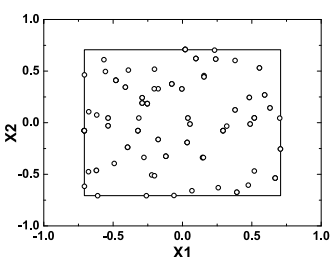

(j)

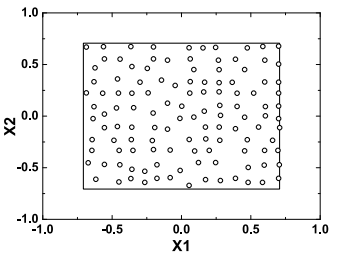

(o)

Fig. 13. Best solution set of the 15 algorithms on a four-objective ML-DMP instance where the search space is $\left[-10^{10}, 10^{10}\right]^{2}$, and its corresponding GD and IGD results. The associated indexes $\left(I_{1}, I_{2}\right)$ of the algorithm, respectively, represent the number of runs (out of all ten runs) in which all obtained solutions converge into (or are close to) the optimal polygon and the number of runs in which the obtained solutions have a good coverage over the optimal polygon. (a) NSGA-II $(0,0) \mathrm{GD}=4.67 \mathrm{E}+9$, IGD $=6.87 \mathrm{E}-1$. (b) SPEA2 $(0,0) \mathrm{GD}=4.34 \mathrm{E}+9$, IGD $=6.85 \mathrm{E}-1$. (c) $\mathrm{AR}(0,0) \mathrm{GD}=2.92 \mathrm{E}+9$, IGD $=$ $4.40 \mathrm{E}+1$. (d) $\operatorname{IBEA}(0,0) \mathrm{GD}=2.76 \mathrm{E}+0$, IGD $=3.50 \mathrm{E}+0$. (e) $\epsilon$-MOEA $(10,0) \mathrm{GD}=0.00 \mathrm{E}+0$, IGD $=5.68 \mathrm{E}-1 .(\mathrm{f}) \mathrm{SMS}-\mathrm{EMOA}(0,0) \mathrm{GD}=1.32 \mathrm{E}+3$, $\mathrm{IGD}=2.94 \mathrm{E}+1 .(\mathrm{g}) \mathrm{MOEA} / \mathrm{D}-\mathrm{TCH}(0,0) \mathrm{GD}=6.99 \mathrm{E}+2, \mathrm{IGD}=7.00 \mathrm{E}+2 .(\mathrm{h}) \mathrm{MOEA} / \mathrm{D}-\mathrm{PBI}(0,0) \mathrm{GD}=2.84 \mathrm{E}+4, \mathrm{IGD}=2.84 \mathrm{E}+4$. (i) $\mathrm{DMO}(0,0)$ $\mathrm{GD}=1.08 \mathrm{E}+9, \mathrm{IGD}=2.19 \mathrm{E}+6 .(\mathrm{j}) \mathrm{HypE}(4,3) \mathrm{GD}=1.08 \mathrm{E}-4$, IGD $=8.90 \mathrm{E}-2$. (k) GrEA $(0,0) \mathrm{GD}=1.40 \mathrm{E}+8$, IGD $=5.29 \mathrm{E}-1$. (1) Two_Arch2 $(0,0) \mathrm{GD}=1.52 \mathrm{E}+9, \mathrm{IGD}=4.63 \mathrm{E}-1 .(\mathrm{m})$ AGE-II $(10,10) \mathrm{GD}=0.00 \mathrm{E}+0, \mathrm{IGD}=5.35 \mathrm{E}-2$. (n) NSGA-III $(0,0)$ GD $=1.00 \mathrm{E}+8$, IGD $=7.77 \mathrm{E}-1$ (o) $\mathrm{SPEA} 2+\mathrm{SDE}(10,10) \mathrm{GD}=0.00 \mathrm{E}+0$, IGD $=5.34 \mathrm{E}-2$.

the low-dimensional instances. They clearly outperform some of the decomposition-based or indicator-based algorithms (e.g., MOEA/D-TCH, MOEA/D-PBI, and IBEA) on the three-objective type III ML-DMP.

2) Due to the lack of diversity maintenance, $A R$ is the algorithm with poor performance on all the instances but the ten-objective type I, where AR is superior to MOEA/D-TCH and HypE in terms of diversity.

3) The performance of IBEA varies, with its solutions distributed well on the type I instances, concentrating into the boundaries of the polygon on the type II instances, and being generally far from the optimal region on the type III instances.

4) $\epsilon$-MOEA performs well on all the types I and II instances, but cannot diversify its solutions on the type III instances. An interesting observation is that $\epsilon$-MOEA struggles to maintain the uniformity on the ten-objective ML-DMP. This is in contrary to some previous studies [19], [68], where the $\epsilon$ dominance has been demonstrated to work well in this respect in the high-dimensional space.

5) SMS-EMOA performs excellently on many relatively easy ML-DMP instances (e.g., on all the three-objective instances). However, when the number of objectives reaches ten, SMS-EMOA fails to provide a good balance between convergence and diversity.
6) MOEA/D-TCH struggles to maintain the uniformity of the solutions over the optimal polygon. This, as explained in [63], is because in the Tchebycheff metric a uniform set of weight vectors may not lead to a uniformly-distributed solutions. In addition, in most cases MOEA/D-TCH cannot guide all of its solutions to converge into the optimal region, although the algorithm performs significantly better than most of the tested algorithms in terms of convergence on the type III instances.

7) The performance of MOEA/D-PBI has a sharp contrast on different instances. It performs perfectly on the three-objective types I and II ML-DMPs, but cannot maintain the uniformity on the other type II instances and promote the convergence on all the type III instances.

8) DMO performs similarly to NSGA-II in most cases. However, due to the adaptive control of the diversity maintenance mechanism, DMO has a better convergence than NSGA-II on some relatively hard ML-DMP instances such as the type II instances with more than three objectives.

9) HypE works fairly well on most of the ML-DMP instances. This includes the two low-dimensional type III ML-DMPs. However, for the ten-objective type I ML-DMP whose search space is precisely the optimal 


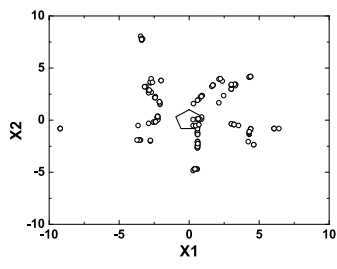

(a)

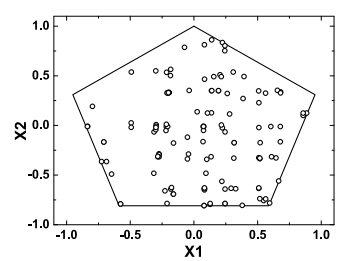

(f)

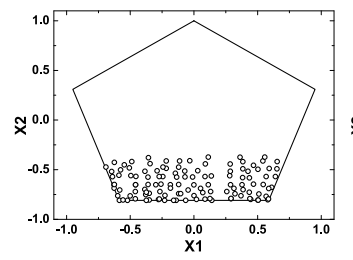

(k)

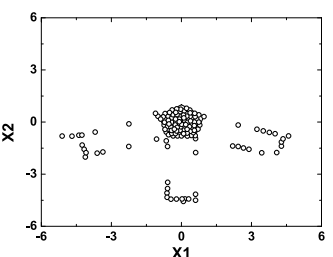

(b)

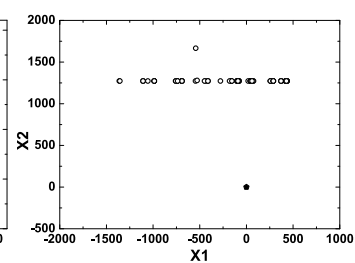

(g)

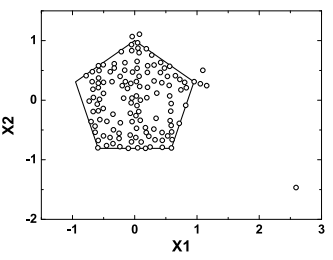

(1)

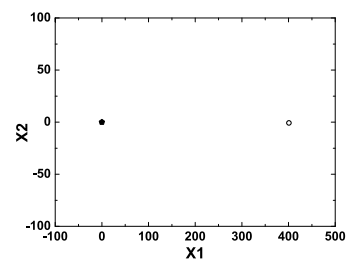

(c)

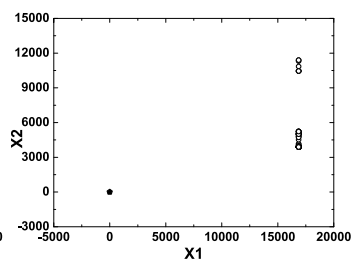

(h)

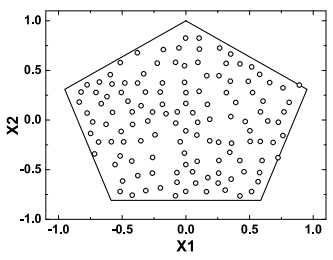

(m)

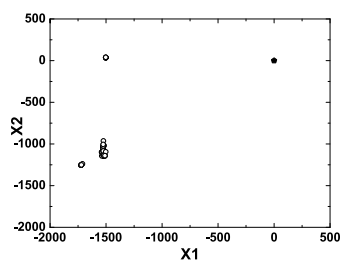

(d)

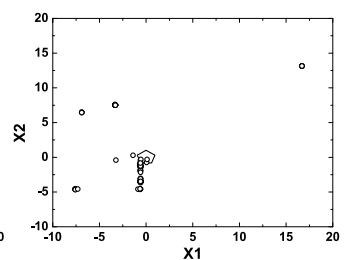

(i)

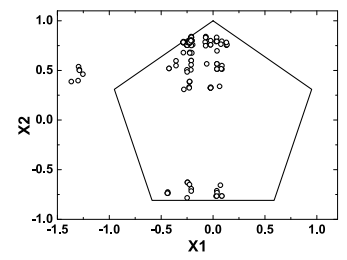

(n)

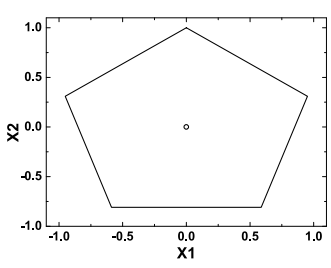

(e)

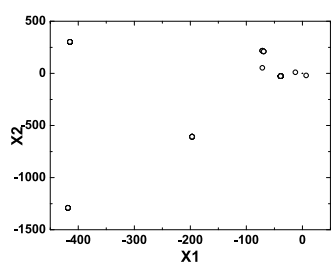

(j)

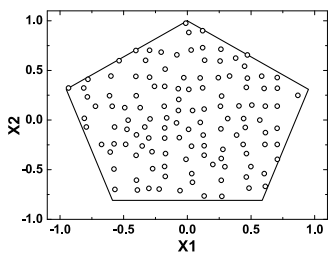

(o)

Fig. 14. Best solution set of the 15 algorithms on a five-objective ML-DMP instance where the search space is $\left[-10^{10} \text {, } 10^{10}\right]^{2}$, and its corresponding GD and IGD results. The associated indexes $\left(I_{1}, I_{2}\right)$ of the algorithm, respectively, represent the number of runs (out of all ten runs) in which all obtained solutions converge into (or are close to) the optimal polygon and the number of runs in which the obtained solutions have a good coverage over the optimal polygon. (a) NSGA-II $(0,0) \mathrm{GD}=5.41 \mathrm{E}+0, \mathrm{IGD}=5.08 \mathrm{E}-1$. (b) $\mathrm{SPEA} 2(0,2) \mathrm{GD}=1.63 \mathrm{E}+0, \mathrm{IGD}=7.22 \mathrm{E}-2$. (c) $\mathrm{AR}(0,0) \mathrm{GD}=4.00 \mathrm{E}+2, \mathrm{IGD}=4.01 \mathrm{E}+2$. (d) $\operatorname{IBEA}(0,0) \mathrm{GD}=1.94 \mathrm{E}+3, \mathrm{IGD}=1.50 \mathrm{E}+3$. (e) $\epsilon$-MOEA $(3,0) \mathrm{GD}=0.00 \mathrm{E}+0$, IGD $=5.97 \mathrm{E}-1$. (f) $\mathrm{SMS}-\mathrm{EMOA}(2,1) \mathrm{GD}=0.00 \mathrm{E}+0, \mathrm{IGD}=$ 8.81E-2. (g) MOEA/D-TCH $(0,0) \mathrm{GD}=4.30 \mathrm{E}+3$, IGD $=2.16 \mathrm{E}+3$. (h) MOEA/D-PBI $(0,0) \mathrm{GD}=1.76 \mathrm{E}+4, \mathrm{IGD}=1.73 \mathrm{E}+4 .(\mathrm{i}) \mathrm{DMO}(0,0) \mathrm{GD}=$ $4.14 \mathrm{E}+0, \mathrm{IGD}=5.12 \mathrm{E}-1$. (j) $\operatorname{HypE}(0,0) \mathrm{GD}=4.33 \mathrm{E}+2, \mathrm{IGD}=1.61 \mathrm{E}+1$. (k) GrEA $(1,0) \mathrm{GD}=0.00 \mathrm{E}+0, \mathrm{IGD}=4.55 \mathrm{E}-1 .(1) \mathrm{Two} \_\mathrm{Arch} 2(0,2) \mathrm{GD}$ $=5.09 \mathrm{E}-2, \mathrm{IGD}=6.60 \mathrm{E}-2 .(\mathrm{m}) \mathrm{AGE}-\mathrm{II}(1,2) \mathrm{GD}=0.00 \mathrm{E}+0, \mathrm{IGD}=5.87 \mathrm{E}-2 .(\mathrm{n}) \mathrm{NSGA}-\mathrm{III}(1,0) \mathrm{GD}=1.80 \mathrm{E}-2, \mathrm{IGD}=3.19 \mathrm{E}-1 .(\mathrm{o}) \mathrm{SPEA} 2+\mathrm{SDE}$ $(5,7) \mathrm{GD}=0.00 \mathrm{E}+0, \mathrm{IGD}=5.88 \mathrm{E}-2$.

polygon, HypE struggles to diversify its solutions over the boundary of the polygon.

10) GrEA performs well on part of the ML-DMP problems. For the three- and five-objective type II instances, GrEA achieves a good performance in terms of both convergence and diversity. For the type III instances with the same dimensions, GrEA can occasionally have a good convergence, but its solutions fail to cover the whole optimal region. For the other types II and III instances, the algorithm cannot lead all of its solutions to converge into the optimal polygon.

11) Due to using the Pareto dominance criterion in the output archive (diversity archive), Two_Arch2 performs similarly to NSGA-II and SPEA2. That is, it works well on all the three-objective instances, but cannot converge when more objectives are involved. However, since the indicator $I_{\epsilon+}$ is used to guide the population in the other archive (convergence archive), Two_Arch2 has a better convergence than the conventional Pareto-based algorithms on some of the many-objective ML-DMPs, such as the five-objective types II and III instances.

12) AGE-II demonstrates its promise on the ML-DMP, with its solutions achieving a good performance in terms of convergence and diversity (esp. uniformity) on most of the tested instances. One exception is the ten-objective type III instance which has a huge search space as well as both parallel target lines and constrained areas.
13) Similar to MOEA/D-PBI, NSGA-III is able to perfectly maintain solutions' diversity for the three-objective MLDMP which has a nondegenerate Pareto front, but struggles on the problem with a degenerate Pareto front. However, NSGA-III shows a clear advantage over MOEA/D-PBI on the three-objective type III instance, but performs worse in terms of convergence for the instances with more objectives. This is probably due to the Pareto dominance criterion which works well on three-objective MOPs but typically fails to provide the selection pressure in a higher-dimensional space.

14) SPEA2+SDE is the only algorithm that is able to obtain a good performance on all the tested instances, despite some not in all the ten runs. Considering both convergence and diversity in its density estimator, SPEA2+SDE can be outperformed by some EMO algorithms in terms of maintaining uniformity on some low-dimensional ML-DMPs, such as SPEA2, $\epsilon$-MOEA, SMS-EMOA, MOEA/D-PBI, Two_Arch2, AGE-II, and NSGA-III. However, this property enables SPEA2+SDE to be promising on those ML-DMPs with the high-dimensional objective space and hard to converge.

Some discussions about the relation between the proposed ML-DMP and existing test problems have been given in Section III of the supplementary material. 


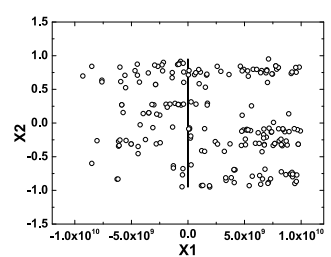

(a)

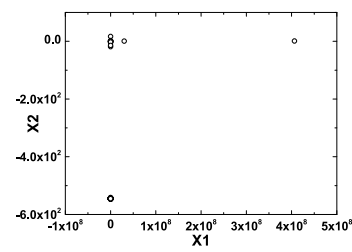

(f)

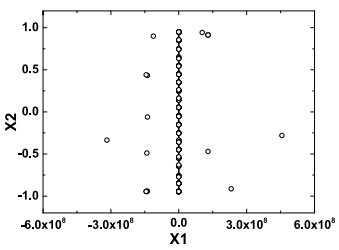

(k)

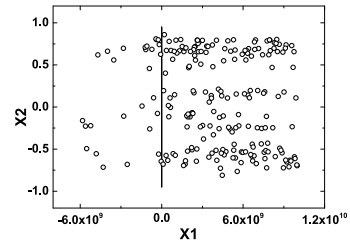

(b)

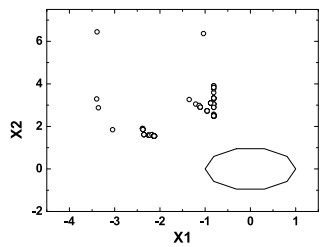

(g)

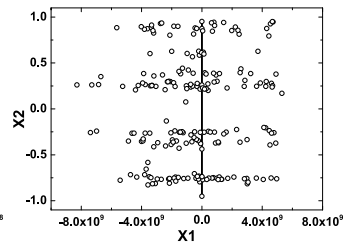

(1)

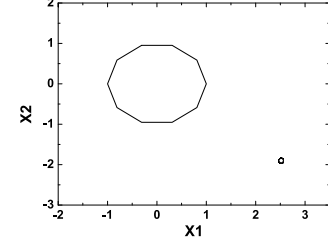

(c)

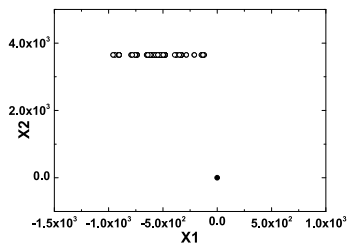

(h)

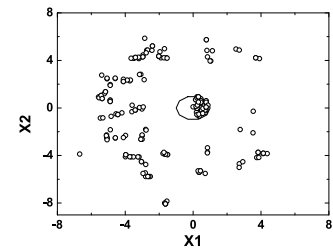

(m)

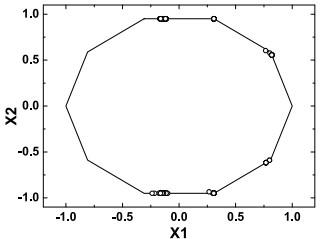

(d)

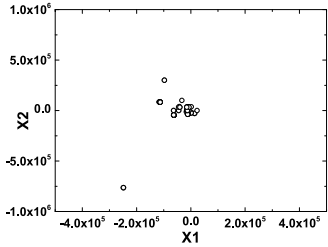

(i)

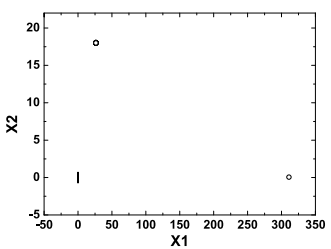

(n)

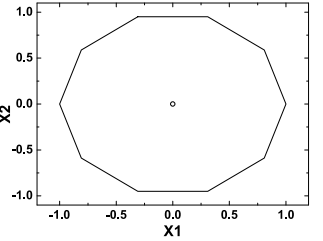

(e)

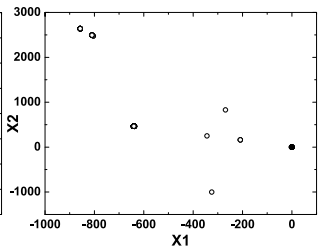

(j)

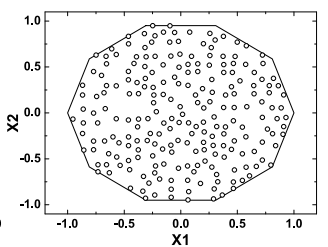

(o)

Fig. 15. Best solution set of the 15 algorithms on a ten-objective ML-DMP instance where the search space is $\left[-10^{10}, 10^{10}\right]^{2}$, and its corresponding GD and IGD results. The associated indexes $\left(I_{1}, I_{2}\right)$ of the algorithm, respectively, represent the number of runs (out of all ten runs) in which all obtained solutions converge into (or are close to) the optimal polygon and the number of runs in which the obtained solutions have a good coverage over the optimal polygon. (a) NSGA-II $(0,0) \mathrm{GD}=4.51 \mathrm{E}+9$, IGD $=1.11 \mathrm{E}+1$. (b) SPEA2 $(0,0) \mathrm{GD}=4.45 \mathrm{E}+9$, IGD $=1.16 \mathrm{E}+1$. (c) $\mathrm{AR}(0,0) \mathrm{GD}=2.16 \mathrm{E}+0, \mathrm{IGD}=3.19 \mathrm{E}+0$. (d) IBEA $(1,0) \mathrm{GD}=0.00 \mathrm{E}+0, \mathrm{IGD}=5.07 \mathrm{E}-1$. (e) $\epsilon$-MOEA $(4,0) \mathrm{GD}=0.00 \mathrm{E}+0$, IGD $=6.60 \mathrm{E}-1$. (f) $\mathrm{SMS}-\mathrm{EMOA}(0,0) \mathrm{GD}=1.98 \mathrm{E}+6, \mathrm{IGD}=$ 8.93E-1. (g) MOEA/D-TCH $(0,0) \mathrm{GD}=3.68 \mathrm{E}+1$, IGD = 2.83E-1. (h) MOEA/D-PBI $(0,0)$ GD = 3.87E+4, IGD = 3.64E+4. (i) DMO $(0,0)$ GD = $4.07 \mathrm{E}+6, \mathrm{IGD}=6.80 \mathrm{E}+3$. (j) $\operatorname{HypE}(0,0) \mathrm{GD}=1.18 \mathrm{E}+3, \mathrm{IGD}=2.62 \mathrm{E}+2$. (k) GrEA $(0,0) \mathrm{GD}=1.13 \mathrm{E}+2, \mathrm{IGD}=9.27 \mathrm{E}-1 .(1) \mathrm{Two}$ Arch2 $(0,0)$ GD $=2.22 \mathrm{E}+9, \mathrm{IGD}=4.42 \mathrm{E}-1 .(\mathrm{m}) \mathrm{AGE}-\mathrm{II}(0,0) \mathrm{GD}=1.46 \mathrm{E}+1, \mathrm{IGD}=1.32 \mathrm{E}-1 .(\mathrm{n}) \mathrm{NSGA}-\mathrm{III}(0,0) \mathrm{GD}=3.22 \mathrm{E}+1, \mathrm{IGD}=3.18 \mathrm{E}+1 .(\mathrm{o}) \mathrm{SPEA} 2+\mathrm{SDE}$ $(4,5) \mathrm{GD}=0.00 \mathrm{E}+0, \mathrm{IGD}=4.97 \mathrm{E}-2$.

\section{CONCLUSION}

This paper presents a class of many-objective test problems, called MLs-DMP, to visually examine EMO algorithms. Fifteen well-established EMO algorithms have been systematically investigated on three types of $10 \mathrm{ML}-\mathrm{DMP}$ instances. Some insights with respect to the design of EMO algorithms have been gained from the investigation, including the followings.

1) Density-based diversity maintenance approaches are suitable for MOPs with a degenerate Pareto front. Indicator-based approaches, as with decompositionbased ones, encounter difficulties in diversifying their population over the degenerate Pareto front.

2) Distinct decomposition-based (or indicator-based) algorithms can behave totally differently. Their combination with Pareto dominance tends to be promising, especially on those low-dimensional MOPs which have a huge search space.

3) Conventional Pareto-based algorithms may completely fail on a four-objective MOP. However, even for manyobjective optimization, it is probably better for EMO algorithms to consider the Pareto dominance criterion first and then another criterion involving both convergence and diversity (or multiple criteria relating to convergence and diversity separately).

4) A small change (relaxation) of the Pareto dominance criterion can be well suited in some many-objective problems. However, this may lead to the loss of the boundaries of the Pareto front to some extent.

The proposed ML-DMP problem differs greatly from the existing ones in the literature. In addition to the geometric similarity, the ML-DMP has the interesting dominance structure which varies with the number of objectives. These characteristics enable it to be a challenge function for EMO algorithms to lead the population toward the Pareto optimal region.

One direction for further study is to extend the number of decision variables of the ML-DMP. A potential way for this can follow the method of the MP-DMP's dimension extension in [34] and [43]. In addition, constructing dynamic ML-DMP problems is also one of our subsequent directions. In this regard, some properties of the ML-DMP can be set to change over time, such as the location of the target lines, the size of the polygon, and even the shape of the polygon (i.e., number of the target lines).

\section{REFERENCES}

[1] H. Ishibuchi, N. Tsukamoto, and Y. Nojima, "Evolutionary manyobjective optimization: A short review," in Proc. IEEE Congr. Evol. Comput. (CEC), Hong Kong, 2008, pp. 2419-2426.

[2] S. Chand and M. Wagner, "Evolutionary many-objective optimization: A quick-start guide," Surveys Oper. Res. Manag. Sci., vol. 20, no. 2, pp. 35-42, 2015.

[3] B. Li, J. Li, K. Tang, and X. Yao, "Many-objective evolutionary algorithms: A survey," ACM Comput. Surveys, vol. 48, no. 1, pp. 1-35, 2015. 
[4] D. J. Walker, R. M. Everson, and J. E. Fieldsend, "Visualizing mutually nondominating solution sets in many-objective optimization," IEEE Trans. Evol. Comput., vol. 17, no. 2, pp. 165-184, Apr. 2013.

[5] K. Miettinen, "Survey of methods to visualize alternatives in multiple criteria decision making problems," OR Spectr., vol. 36, no. 1, pp. 3-37, 2014.

[6] A. R. R. de Freitas, P. J. Fleming, and F. G. Guimarães, "Aggregation Trees for visualization and dimension reduction in many-objective optimization," Inf. Sci., vol. 298, pp. 288-314, Mar. 2015.

[7] T. Tušar and B. Filipič, "Visualization of Pareto front approximations in evolutionary multiobjective optimization: A critical review and the prosection method," IEEE Trans. Evol. Comput., vol. 19, no. 2, pp. 225-245, Apr. 2015.

[8] Z. He and G. G. Yen, "Visualization and performance metric in manyobjective optimization," IEEE Trans. Evol. Comput., vol. 20, no. 3, pp. 386-402, Jun. 2016.

[9] K. Deb, L. Thiele, M. Laumanns, and E. Zitzler, "Scalable test problems for evolutionary multiobjective optimization," in Evolutionary Multiobjective Optimization. Theoretical Advances and Applications, A. Abraham, L. Jain, and R. Goldberg, Eds. Berlin, Germany: Springer, 2005, pp. 105-145.

[10] S. Huband, P. Hingston, L. Barone, and L. While, "A review of multiobjective test problems and a scalable test problem toolkit," IEEE Trans. Evol. Comput., vol. 10, no. 5, pp. 477-506, Oct. 2006.

[11] E. Zitzler and L. Thiele, "Multiobjective evolutionary algorithms: A comparative case study and the strength Pareto approach," IEEE Trans. Evol. Comput., vol. 3, no. 4, pp. 257-271, Nov. 1999.

[12] D. W. Corne and J. D. Knowles, "Techniques for highly multiobjective optimisation: Some nondominated points are better than others," in Proc. 9th Annu. Conf. Genet. Evol. Comput. (GECCO), London, U.K., 2007, pp. 773-780.

[13] H. E. Aguirre and K. Tanaka, "Working principles, behavior, and performance of MOEAs on MNK-landscapes," Eur. J. Oper. Res., vol. 181, no. 3, pp. 1670-1690, 2007.

[14] D. K. Saxena, Q. Zhang, J. A. Duro, and A. Tiwari, "Framework for many-objective test problems with both simple and complicated Pareto-set shapes," in Proc. Evol. Multi Criterion Optim. (EMO), Ouro Preto, Brazil, 2011, pp. 197-211.

[15] Y.-M. Cheung, F. Gu, and H.-L. Liu, "Objective extraction for manyobjective optimization problems: Algorithm and test problems," IEEE Trans. Evol. Comput., vol. 20, no. 5, pp. 755-772, Oct. 2016.

[16] R. Cheng, Y. Jin, M. Olhofer, and B. Sendhoff, "Test problems for largescale multiobjective and many-objective optimization," IEEE Trans. Cybern., to be published.

[17] T. Wagner, N. Beume, and B. Naujoks, "Pareto-, aggregation-, and indicator-based methods in many-objective optimization," in Proc. Evol. Multi Criterion Optim. (EMO), Matsushima, Japan, 2007, pp. 742-756.

[18] A. L. Jaimes and C. A. C. Coello, "Study of preference relations in many-objective optimization," in Proc. 11th Annu. Conf. Genet. Evol. Comput. (GECCO), Montreal, QC, Canada, 2009, pp. 611-618.

[19] D. Hadka and P. Reed, "Diagnostic assessment of search controls and failure modes in many-objective evolutionary optimization," Evol. Comput., vol. 20, no. 3, pp. 423-452, Sep. 2012.

[20] R. Wang, R. C. Purshouse, and P. J. Fleming, "Preference-inspired coevolutionary algorithms for many-objective optimization," IEEE Trans. Evol. Comput., vol. 17, no. 4, pp. 474-494, Aug. 2013.

[21] Z. He, G. G. Yen, and J. Zhang, "Fuzzy-based Pareto optimality for many-objective evolutionary algorithms," IEEE Trans. Evol. Comput., vol. 18, no. 2, pp. 269-285, Apr. 2014

[22] M. Li, S. Yang, and X. Liu, "Bi-goal evolution for many-objective optimization problems," Artif. Intell., vol. 228, pp. 45-65, Nov. 2015.

[23] X. Zhang, Y. Tian, and Y. Jin, "A knee point-driven evolutionary algorithm for many-objective optimization," IEEE Trans. Evol. Comput. vol. 19, no. 6, pp. 761-776, Dec. 2015.

[24] K. Li, K. Deb, Q. Zhang, and S. Kwong, "An evolutionary manyobjective optimization algorithm based on dominance and decomposition," IEEE Trans. Evol. Comput., vol. 19, no. 5, pp. 694-716, Oct. 2015

[25] R. Cheng, Y. Jin, M. Olhofer, and B. Sendhoff, "A reference vector guided evolutionary algorithm for many-objective optimization," IEEE Trans. Evol. Comput., vol. 20, no. 5, pp. 773-791, Oct. 2016.

[26] Y. Xiang, Y. Zhou, M. Li, and Z. Chen, "A vector angle based evolutionary algorithm for unconstrained many-objective optimization," IEEE Trans. Evol. Comput., to be published.

[27] M. Li, S. Yang, and X. Liu, "A performance comparison indicator for Pareto front approximations in many-objective optimization," in Proc. 17th Conf. Genet. Evol. Comput. (GECCO), Madrid, Spain, 2015, pp. $703-710$.
[28] C. A. C. Coello and M. R. Sierra, "A study of the parallelization of a coevolutionary multi-objective evolutionary algorithm," in Proc. Mex. Int. Conf. Artif. Intell. (MICAI), Mexico City, Mexico, 2004, pp. 688-697.

[29] S. Jiang, Y.-S. Ong, J. Zhang, and L. Feng, "Consistencies and contradictions of performance metrics in multiobjective optimization," IEEE Trans. Cybern., vol. 44, no. 12, pp. 2391-2404, Dec. 2014.

[30] H. Ishibuchi, H. Masuda, and Y. Nojima, "Pareto fronts of manyobjective degenerate test problems," IEEE Trans. Evol. Comput., vol. 20, no. 5, pp. 807-813, Oct. 2016

[31] M. Li, S. Yang, and X. Liu, "Pareto or non-Pareto: Bi-criterion evolution in multiobjective optimization," IEEE Trans. Evol. Comput., vol. 20, no. 5, pp. 645-665, Oct. 2016.

[32] M. Köppen, R. Vicente-Garcia, and B. Nickolay, "The Pareto-box problem for the modelling of evolutionary multiobjective optimization algorithms," in Adaptive and Natural Computing Algorithms. Vienna, Austria: Springer, 2005, pp. 194-197.

[33] M. Köppen and K. Yoshida, "Substitute distance assignments in NSGA-II for handling many-objective optimization problems," in Proc. Evol. Multi Criterion Optim. (EMO), Matsushima, Japan, 2007, pp. $727-741$.

[34] H. Ishibuchi, M. Yamane, N. Akedo, and Y. Nojima, "Many-objective and many-variable test problems for visual examination of multiobjective search," in Proc. IEEE Congr. Evol. Comput. (CEC), Cancún, Mexico, 2013, pp. 1491-1498.

[35] H. Zille and S. Mostaghim, "Properties of scalable distance minimization problems using the Manhattan metric," in Proc. IEEE Congr. Evol. Comput. (CEC), Sendai, Japan, 2015, pp. 2875-2882.

[36] J. Xu, K. Deb, and A. Gaur, "Identifying the Pareto-optimal solutions for multi-point distance minimization problem in Manhattan space," Comput. Optim. Innov. (COIN) Lab., East Lansing, MI, USA, COIN Tech. Rep. 2015018, 2015.

[37] R. G. Karlsson and M. H. Overmars, "Scanline algorithms on a grid," BIT Numer. Math., vol. 28, no. 2, pp. 227-241, 1988.

[38] G. Rudolph, B. Naujoks, and M. Preuss, "Capabilities of EMOA to detect and preserve equivalent Pareto subsets," in Proc. Evol. Multi Criterion Optim. (EMO), Matsushima, Japan, 2007, pp. 36-50.

[39] O. Schütze, A. Lara, and C. A. C. Coello, "On the influence of the number of objectives on the hardness of a multiobjective optimization problem," IEEE Trans. Evol. Comput., vol. 15, no. 4, pp. 444-455, Aug. 2011.

[40] H. K. Singh, A. Isaacs, T. Ray, and W. Smith, "A study on the performance of substitute distance based approaches for evolutionary many objective optimization," in Proc. Simulat. Evol. Learn. (SEAL), Melbourne, VIC, Australia, 2008, pp. 401-410.

[41] H. Ishibuchi, Y. Hitotsuyanagi, N. Tsukamoto, and Y. Nojima, "Many-objective test problems to visually examine the behavior of multiobjective evolution in a decision space," in Proc. Int. Conf. Parallel Problem Solving Nat. (PPSN), Kraków, Poland, 2010, pp. 91-100.

[42] H. Ishibuchi, N. Akedo, and Y. Nojima, "A many-objective test problem for visually examining diversity maintenance behavior in a decision space," in Proc. 13th Annu. Conf. Genet. Evol. Comput. (GECCO), Dublin, Ireland, 2011, pp. 649-656.

[43] H. Masuda, Y. Nojima, and H. Ishibuchi, "Visual examination of the behavior of EMO algorithms for many-objective optimization with many decision variables," in Proc. IEEE Congr. Evol. Comput. (CEC), Beijing, China, 2014, pp. 2633-2640.

[44] J. E. Fieldsend, "Enabling dominance resistance in visualisable distancebased many-objective problems," in Proc. Genet. Evol. Comput. Conf. Companion (GECCO), Denver, CO, USA, 2016, pp. 1429-1436.

[45] M. Li, S. Yang, X. Liu, and R. Shen, "A comparative study on evolutionary algorithms for many-objective optimization," in Proc. Evol. Multi Criterion Optim. (EMO), Sheffield, U.K., 2013, pp. 261-275.

[46] M. Li, S. Yang, and X. Liu, "Shift-based density estimation for Paretobased algorithms in many-objective optimization," IEEE Trans. Evol. Comput., vol. 18, no. 3, pp. 348-365, Jun. 2014.

[47] K. Narukawa, "Finding a diverse set of decision variables in evolutionary many-objective optimization," in Proc. 15th Annu. Conf. Companion Genet. Evol. Comput., Amsterdam, The Netherlands, 2013, pp. 95-96.

[48] M. Li, S. Yang, and X. Liu, "A test problem for visual investigation of high-dimensional multi-objective search," in Proc. IEEE Congr. Evol. Comput. (CEC), Beijing, China, 2014, pp. 2140-2147.

[49] K. Ikeda, H. Kita, and S. Kobayashi, "Failure of Pareto-based MOEAs: Does non-dominated really mean near to optimal?" in Proc. IEEE Congr. Evol. Comput. (CEC), vol. 2. Seoul, South Korea, 2001, pp. 957-962. 
[50] A. L. Jaimes, "Techniques to deal with many-objective optimization problems using evolutionary algorithms," Ph.D. dissertation, Comput. Sci. Dept., Center Res. Adv. Stud. Nat. Polytech. Inst. Mexico, Mexico City, Mexico, May 2011.

[51] K. Deb, A. Pratap, S. Agarwal, and T. Meyarivan, "A fast and elitist multiobjective genetic algorithm: NSGA-II," IEEE Trans. Evol. Comput., vol. 6, no. 2, pp. 182-197, Apr. 2002.

[52] E. Zitzler, M. Laumanns, and L. Thiele, "SPEA2: Improving the strength Pareto evolutionary algorithm for multiobjective optimization," in Proc. Evol. Methods Design Optim. Control, 2002, pp. 95-100.

[53] P. J. Bentley and J. P. Wakefield, "Finding acceptable solutions in the Pareto-optimal range using multiobjective genetic algorithms," in Soft Computing in Engineering Design and Manufacturing. London, U.K.: Springer, 1997, ch. 5, pp. 231-240.

[54] E. Zitzler and S. Künzli, "Indicator-based selection in multiobjective search," in Proc. Int. Conf. Parallel Problem Solving Nat. (PPSN), Birmingham, U.K., 2004, pp. 832-842.

[55] K. Deb, M. Mohan, and S. Mishra, "Evaluating the $\epsilon$-domination based multi-objective evolutionary algorithm for a quick computation of Pareto-optimal solutions," Evol. Comput., vol. 13, no. 4, pp. 501-525, Dec. 2005

[56] N. Beume, B. Naujoks, and M. Emmerich, "SMS-EMOA: Multiobjective selection based on dominated hypervolume," Eur. J. Oper. Res., vol. 181 no. 3, pp. 1653-1669, 2007.

[57] Q. Zhang and H. Li, "MOEA/D: A multiobjective evolutionary algorithm based on decomposition," IEEE Trans. Evol. Comput., vol. 11, no. 6, pp. 712-731, Dec. 2007.

[58] S. F. Adra and P. J. Fleming, "Diversity management in evolutionary many-objective optimization," IEEE Trans. Evol. Comput., vol. 15, no. 2, pp. 183-195, Apr. 2011.

[59] J. Bader and E. Zitzler, "HypE: An algorithm for fast hypervolume-based many-objective optimization," Evol. Comput., vol. 19, no. 1, pp. 45-76, Mar. 2011

[60] S. Yang, M. Li, X. Liu, and J. Zheng, "A grid-based evolutionary algorithm for many-objective optimization," IEEE Trans. Evol. Comput., vol. 17, no. 5, pp. 721-736, Oct. 2013.

[61] H. Wang, L. Jiao, and X. Yao, "Two_Arch2: An improved two-archive algorithm for many-objective optimization," IEEE Trans. Evol. Comput., vol. 19, no. 4, pp. 524-541, Aug. 2015.

[62] M. Wagner and F. Neumann, "A fast approximation-guided evolutionary multi-objective algorithm," in Proc. 15th Annu. Conf. Genet. Evol. Comput. (GECCO), Amsterdam, The Netherlands, 2013, pp. 687-694.

[63] K. Deb and H. Jain, "An evolutionary many-objective optimization algorithm using reference-point-based nondominated sorting approach, part I: Solving problems with box constraints," IEEE Trans. Evol. Comput., vol. 18, no. 4, pp. 577-601, Aug. 2014.

[64] M. Li, J. Zheng, K. Li, Q. Yuan, and R. Shen, "Enhancing diversity for average ranking method in evolutionary many-objective optimization," in Proc. Int. Conf. Parallel Problem Solving Nat. (PPSN), Kraków, Poland, 2010, pp. 647-656.

[65] M. Laumanns, L. Thiele, K. Deb, and E. Zitzler, "Combining convergence and diversity in evolutionary multiobjective optimization," Evol. Comput., vol. 10, no. 3, pp. 263-282, Sep. 2002.

[66] K. Bringmann, T. Friedrich, F. Neumann, and M. Wagner, "Approximation-guided evolutionary multi-objective optimization," in Proc. 22nd Int. Joint Conf. Artif. Intell. (IJCAI), Barcelona, Spain, 2011, pp. 1198-1203.

[67] D. A. Van Veldhuizen and G. B. Lamont, "Evolutionary computation and convergence to a Pareto front," in Proc. Late Breaking Papers Genet. Program. Conf., 1998, pp. 221-228.

[68] D. Hadka and P. Reed, "Borg: An auto-adaptive many-objective evolutionary computing framework," Evol. Comput., vol. 21, no. 2 , pp. 231-259, May 2013.

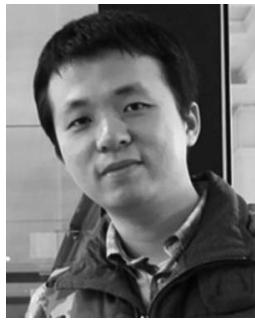

Miqing Li received the Ph.D. degree in computer science from the Department of Computer Science, Brunel University London, Uxbridge, U.K., in 2015.

$\mathrm{He}$ is currently a Research Fellow with the Centre of Excellence for Research in Computational Intelligence and Applications, School of Computer Science, University of Birmingham, Birmingham, U.K. His current research interests include evolutionary multiobjective and many-objective optimization, including algorithm design, performance assessment, test problem construction and visualization, and their application in diverse fields.

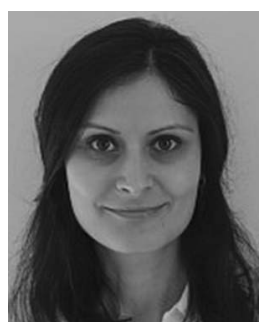

Crina Grosan received the B.Sc. degree in mathematics and computer science, the M.Sc. degree in complex analysis and optimization, and the Ph.D. degree in artificial intelligence from Babeş-Bolyai University, Cluj-Napoca, Romania

She is a Lecturer with the Department of Computer Science, College of Engineering, Design and Physical Sciences, Brunel University London, Uxbridge, U.K. Her current research interests include the development of artificial intelligence/machine learning methods and algorithms for large-scale optimization, systems of equations, data analysis, graphs and decision making, with applications in medicine, biology, pharmacy, economics, and engineering.

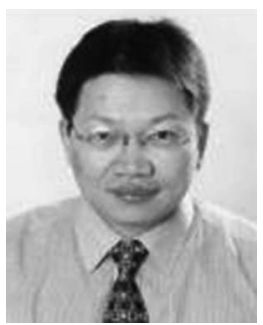

Shengxiang Yang (M'00-SM'14) received the B.Sc. and M.Sc. degrees in automatic control and the $\mathrm{Ph} . \mathrm{D}$. degree in systems engineering from Northeastern University, Shenyang, China, in 1993 , 1996, and 1999, respectively.

$\mathrm{He}$ is currently a Professor of Computational Intelligence and the Director of the Centre for Computational Intelligence, School of Computer Science and Informatics, De Montfort University, Leicester, U.K. He has over 220 publications. His current research interests include evolutionary and genetic algorithms, swarm intelligence, computational intelligence in dynamic and uncertain environments, artificial neural networks for scheduling, and relevant real-world applications.

Dr. Yang serves as an Associate Editor or an Editorial Board Member for eight international journals, such as the IEEE TRANSACTIONS ON CYBERNETICS, Information Sciences, Evolutionary Computation, Neurocomputing, and Soft Computing.

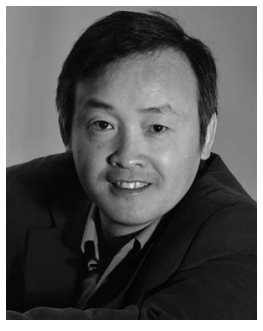

Xiaohui Liu received the B.Eng. in computing from Hohai University, Nanjing, China, and a Ph.D. in computer science from Heriot-Watt University, Edinburgh, U.K.

$\mathrm{He}$ is a Professor of Computing with Brunel University London, Uxbridge, U.K., where he directs the Centre for Intelligent Data Analysis. He has over 100 journal publications in computational intelligence and data science.

Prof. Liu was a recipient of the Highly Cited Researchers Award by Thomson Reuters.

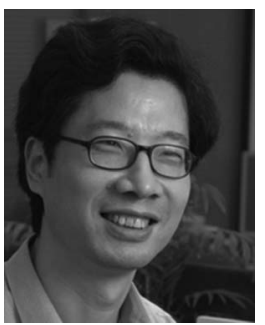

Xin Yao (F'03) is a Chair Professor of Computer Science with the Southern University of Science and Technology, Shenzhen, China, and a Professor of Computer Science with the University of Birmingham, Birmingham, U.K. He has been researching multiobjective optimization since 2003 , when he published a well-cited EMO'03 paper on many-objective optimization. His current research interests include evolutionary computation, ensemble learning, and their applications in software engineering.

Prof. Yao was a recipient of the 2001 IEEE Donald G. Fink Prize Paper Award, the 2010, 2016, and 2017 IEEE TRANSACTIONS ON Evolutionary COMPUTATion Outstanding Paper Awards, the 2010 BT Gordon Radley Award for Best Author of Innovation (Finalist), the 2011 IEEE Transactions on Neural Networks Outstanding Paper Award, the Prestigious Royal Society Wolfson Research Merit Award in 2012, the IEEE Computational Intelligence Society (CIS) Evolutionary Computation Pioneer Award in 2013, and several other best paper awards. He was the President of the IEEE CIS from 2014 to 2015, and the Editor-in-Chief of the IEEE TRANSACTIONS ON EVOLUTIONARY COMPUTATION from 2003 to 2008. He is a Distinguished Lecturer of the IEEE CIS 